\title{
Reliable and Sophisticated Advanced Stressed Crises Compound Options Pricing Models
}

\author{
Jamilu Auwalu Adamu, ${ }^{1, *}$ \\ ${ }^{1}$ Mathematics Programme, National Mathematical Centre, Abuja, Nigeria \\ *Correspondence: Mathematics Programme, National Mathematical Centre, Abuja, Nigeria. Tel: 234-80-3867-9094. \\ E-mail: whitehorseconsult@yahoo.com
}

Received: September 23, 2015

doi:10.5430/mos.v3n1p39
Accepted: October 21, 2015 Online Published: January 9, 2016

URL: http://dx.doi.org/10.5430/mos.v3n1p39

\begin{abstract}
Compound Options are Exotic Derivatives Instruments that can be used to minimize the degree of investment risk, in hedging and speculative strategies which makes them Cheaper than the plain vanilla options and highly leveraged.From the traditional Robert Geske (1979) and Rubistein (1991) methods of compound options valuation (which simply assumed Normality) to Monte Carlo simulations method of valuation (which transform $\mathrm{n}-$ simulations of uniform variables into Normal Variables) were all underestimate (overestimate) the compound options prices because of the simple Normality Assumptions that governed the formulation of the models or process.Above all they lag the incorporation of Fat - Tailed Effects of returns on compound options underlying assets' probability distributions to enable them capture the popular Black Swan Events as propagated by Nassim N. Taleb and other professional risk management associations like PRMIA.However, in this paper, the Author attempted to incorporate fat - tailed effects into the models using Jameel's Contractional and Expansional Stress Methods so as to enable them precisely traces the trajectories of the Past and Future Black Swan events to avoid reoccurrences of future economic and financial crises related to exotic options more often otherwise makes the existing models more reliable, robust, sophisticated and holistic. Finally, the proposed Jameel's Advanced Stressed Exotic Options Pricing Models are expected to dramatically increase the markets Confidence and drastically decreases the markets Risks.
\end{abstract}

Keywords: Strike; Exotic; Fat-tailed; Call; Put; Jameel

\section{Introduction}

The Compound Option gives the holder the right to buy (in case of Call) or sell (in case of Put) the underlying Option. Compound options are options on options. The underlying asset is itself an Option.Compound Option has two strike prices ( $X_{1}$ : Compound and $X_{2}$ : underlying) and two expiration dates ( $T_{1}$ : Compound and $T_{2}$ : underlying with $\left.T_{1}<T_{2}\right)$. At the first exercise date $T_{1}$ you must decide whether it is worth exercising the first option (depending on the strike price $X_{1}$ and the current asset price S). If so, you get a further option with strike price $X_{2}$ and maturity $T_{2}$. There are four basic types of compound options: Call on Call (CoC), Call on Put (CoP) or caput option, Put on Put (PoP) and Put on Call (PoC). The payoff of a compound option involves the value of another option.There are four possible payoffs: Call on call: $\operatorname{Max}\left\{C\left(S, T_{1}\right)-X_{1}, 0\right\}$, Call on put: $\operatorname{Max}\left\{P\left(S, T_{1}\right)-X_{1}, 0\right\}$, Put on call: $\operatorname{Max}\left\{X_{1}-C\left(S, T_{1}\right), 0\right\}$ and Put on put: $\operatorname{Max}\left\{X_{1}-P\left(S, T_{1}\right), 0\right\}$.

Robert Geske (1979) and Rubistein (1991) were the first to find the solution and implement the closed form formulae on the basis of the Black - Scholes - Merton Model (1973) to price European - type of compound options. Other research has been conducted by Thomassen and Van Wouwe (2002).

The overreliance of NORMALITY ASSUMPTIONS in the valuation process of Exotic Options (Compound 
Options) has seriously and continuously threatens the resilient of economic and financial stability reference to the Exotic Options Pricing.Tremendously,the Small Probabilities Margin has significant IMPACT in our Exotic (Compound) Options Prices as propagated by Nassim N. Taleb and other world economic and financial leading actors. The Robert Geske (1979) and Rubistein (1991) method of valuing compound options assumed normality and constant volatility, so they out rightly underestimates the prices while the Monte Carlo simulations transform $\mathrm{n}-$ simulations of uniform variables into normal variables, however, these seriously NEGLECTED the potential effects of Small Probabilities Margin or Low - Probability, High - Impact events occurs at the fourth quadrant . These are of course thrown the risk of underestimating (overestimating) compound options prices to the entire world economic and financial systems.

In view of the foregoing, we need to EXPLICITLY STRESS all our Exotic Options Pricing Models by incorporating fat - tailed effects into them to enable them precisely capture potential Black Swans.

In this paper, the Author consider Robert Geske (1979) and Rubistein (1991) compound options pricing models and incorporates fat - tailed effects into them using Jameel'sContractional and Expansional Stress Methods.

\section{Material and Method}

2.1 Robert Geske (1979) - Rubistein (1991) Assumptions and Monte Carlo Simulations Method of Valuing Compound Options

2.1.1 Jameel's Observations

- Robert Geske (1979) and Rubinstein (1991) assumed NORMALITY and CONSTANT VOLATILITY because of this, the price obtained with the models are generally UNDERESTIMATED.

- The Monte Carlo Simulations Model implements $\mathrm{n}$ - Simulations of uniform variables which then transforms into NORMAL variables.

- The FAT - TAILED EFFECTS on the RETURNS of the underlying probability distributions of $S, X_{1}$ and $X_{2}$ are NOT considered in both Robert Geske (1979), Rubistein (1991) and Monte Carlo Simulations Method.

- After the Stock market Crash of 1987, the concepts of VOLITILITY SMILE and SKEW came into account. That is fat - tailed effects in the underlying assets distributions. This concept critically analyses the presence of SKEW and HEAVY KUTOSIS in the options strikes prices over different volatilities. However, the existing models do not take into account the incorporation of volatility smile and skew.

- Furthermore, there is concrete evidence that the empirical returns distribution DOES NOT appear to be GAUSSIAN.

- Hence, there are great tendencies of the Compound (Exotic) Options Models to Underestimate (Overestimate) the prices especially at the times of economic recoveries or recessions.

That is why Jameel'sContractional and ExpansionalStress Methods addresses the above Observations as follows.

\subsection{Method}

The methodology adopted in this research work is to use Jameel'sContractional and Expansional stress methods to

SEVERELY stress the traditional Compound (Exotic) Options Pricing Models such as Robert Geske (1979), its

Greeks, and Rubistein (1991) using (a) Geometric Return of the Arithmetic Means of the U.S. macroeconomic indicators plus research company underlying stock returns $\mu_{S}$ and the Geometric volatility of the volatilities of the U.S. macroeconomic indicators plus research company underlying stock returns $\sigma_{S}$ as well as the Best fitted fat tailed probability distribution of the research company underlying stock returns $f_{3}\left(s, \mu_{S}, \sigma_{S}, \xi\right)$ (b) Geometric Return of the Arithmetic Means of the U.S. macroeconomic indicators plus research company simple underlying strike returns $\mu_{X_{2}}$ and the Geometric volatility of the volatilities of the U.S. macroeconomic indicators plus research company simple underlying strike returns $\sigma_{X_{2}}$ (c) Geometric Return of the Arithmetic Means of the U.S. 
macroeconomic indicators plus research company compound underlying strike returns $\mu_{X_{1}}$ and the Geometric volatility of the volatilities of the U.S. macroeconomic indicators plus research company compound underlying strike returns $\sigma_{X_{1}}$ as well as the Best fitted fat - tailed probability distribution of the research companysimple and compound underlying strikesreturns $f_{2}\left(x_{2}, \mu, \sigma, \pi\right)$ and $f_{1}\left(x_{1}, \mu, \sigma, \pi\right)$ (which are the BEST FITTEDFat - Tailed Probability Distributions of the Compound Options' Two Strikes obtained using Jameel's Criterion) respectively (c) Geometric Return of the Arithmetic Means of the U.S. macroeconomic indicators plus research company underlying stock returns and underlying two strikesreturns $\mu_{S_{X_{1} X_{2}}}$ and the Geometric volatility of the volatilities of the U.S. macroeconomic indicators plus research company underlying stock returns and underlying two strikesreturns $\sigma_{S X_{1} X_{2}}$ given that $X_{1}$ : is the underlying compound option Strike Price and $X_{2}$ : is the simple underlying Strike Price as shown below:

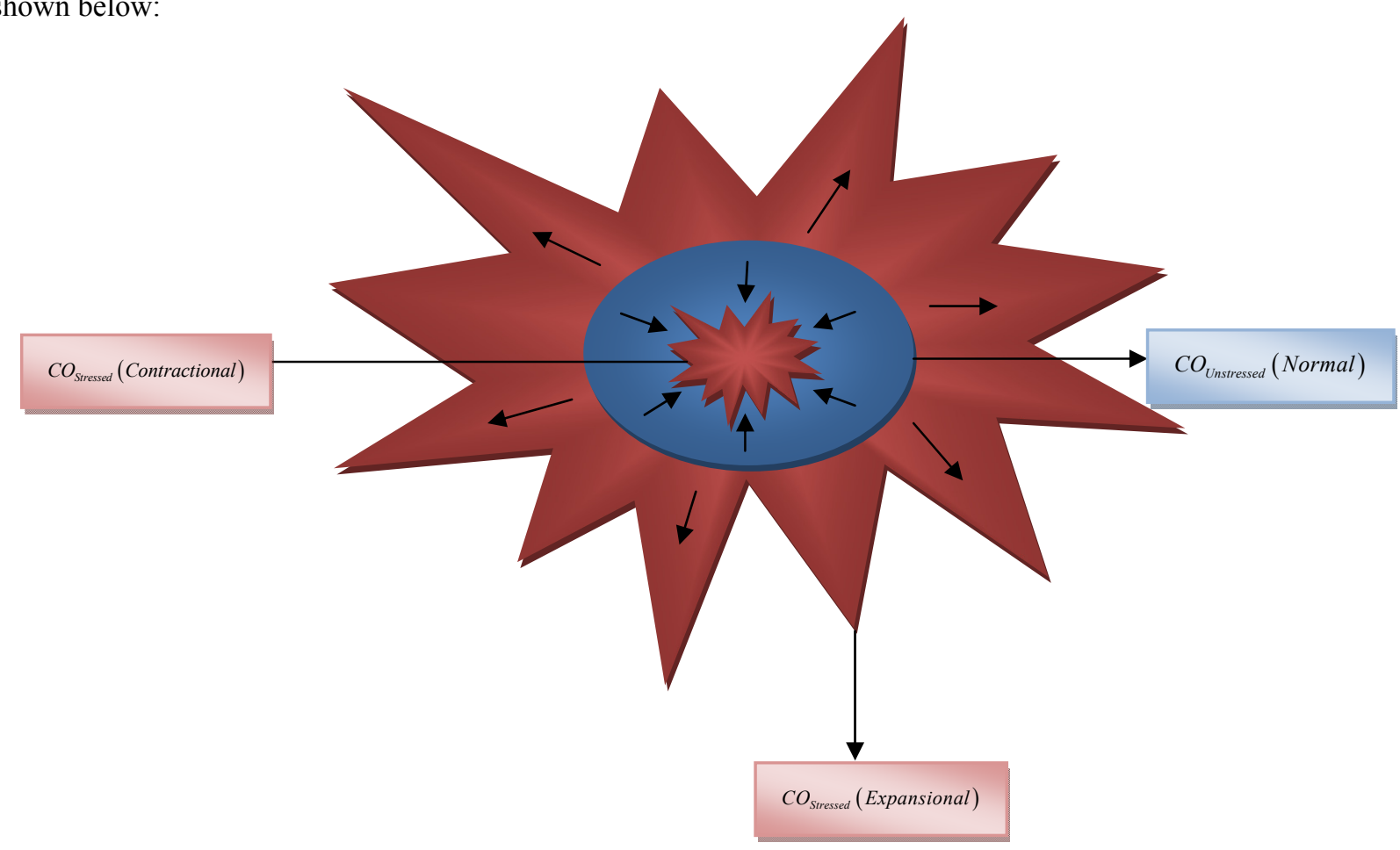

Figure 1. Jameel's Contractional - Expansional Stress Diagram

\subsubsection{Proposed Jameel's CALL on CALL Stressed Option Models}

\section{Class 1:}

$$
\begin{aligned}
C C_{\text {Stressed }}= & S e^{-q T_{2}}\left[N_{2}\left(\mu_{X_{1}} \mu_{X_{2}} \mu_{S}\left(a_{1}, b_{1} ; \sqrt{T_{1} / T_{2}}\right)\right) \pm \sigma_{X_{1}} f_{1}(.) \pm \sigma_{X_{2}} f_{2}(.) \pm \sigma_{S} f_{3}(.)\right] \\
& -X_{2} e^{-r T_{2}}\left[N_{2}\left(\mu_{X_{1}} \mu_{X_{2}} \mu_{S}\left(a_{2}, b_{2} ; \sqrt{T_{1} / T_{2}}\right)\right) \pm \sigma_{X_{1}} f_{1}(.) \pm \sigma_{X_{2}} f_{2}(.) \pm \sigma_{S} f_{3}(.)\right] \\
& -X_{1} e^{-r T_{1}}\left[N\left(\mu_{X_{1}} \mu_{X_{2}} \mu_{S}\left(a_{2}\right)\right) \pm \sigma_{X_{1}} f_{1}(.) \pm \sigma_{X_{2}} f_{2}(.) \pm \sigma_{S} f_{3}(.)\right]
\end{aligned}
$$

\section{Class 2:}




$$
\begin{aligned}
C C_{\text {Stressed }}= & S e^{-q T_{2}}\left[N_{2}\left(\mu_{X_{1} X_{2} s}\left(a_{1}, b_{1} ; \sqrt{T_{1} / T_{2}}\right)\right) \pm \sigma_{X_{1} X_{2} S} f_{1}(.) \pm \sigma_{X_{1} X_{2} S} f_{2}(.) \pm \sigma_{X_{1} X_{2} S} f_{3}(.)\right] \\
& -X_{2} e^{-r T_{2}}\left[N_{2}\left(\mu_{X_{1} X_{2} s}\left(a_{2}, b_{2} ; \sqrt{T_{1} / T_{2}}\right)\right) \pm \sigma_{X_{1} X_{2} s} f_{1}(.) \pm \sigma_{X_{1} X_{2} S} f_{2}(.) \pm \sigma_{X_{1} X_{2} s} f_{3}(.)\right] \\
& -X_{1} e^{-r T_{1}}\left[N\left(\mu_{X_{1} X_{2} S}\left(a_{2}\right)\right) \pm \sigma_{X_{1} X_{2} S} f_{1}(.) \pm \sigma_{X_{1} X_{2} S} f_{2}(.) \pm \sigma_{X_{1} X_{2} S} f_{3}(.)\right]
\end{aligned}
$$

Where,

$$
\begin{aligned}
C C=S e^{-q T_{2}} & N_{2}\left(a_{1}, b_{1} ; \sqrt{T_{1} / T_{2}}\right) \\
& -X_{2} e^{-r T_{2}} N_{2}\left(a_{2}, b_{2} ; \sqrt{T_{1} / T_{2}}\right) \\
& -X_{1} e^{-r T_{1}} N\left(a_{2}\right)
\end{aligned}
$$

These are the general FORMS of the proposal, however, we can use COMBINATORICS to obtain different possible Combination of TERMS and SIGNS (to achieve different values of $C C_{\text {Stressed }}$ : positive, negative, high, low, close and so on).CLASS 1 and 2 are OPTIMUM at:

$$
\begin{aligned}
C C_{\text {Stressed }}= & S e^{-q T_{2}}\left[N_{2}\left(a_{1}, b_{1} ; \sqrt{T_{1} / T_{2}}\right) \pm \sigma_{X_{1}} f_{1}(.) \pm \sigma_{X_{2}} f_{2}(.) \pm \sigma_{S} f_{3}(.)\right] \\
& -X_{2} e^{-r T_{2}}\left[N_{2}\left(a_{2}, b_{2} ; \sqrt{T_{1} / T_{2}}\right) \pm \sigma_{X_{1}} f_{1}(.) \pm \sigma_{X_{2}} f_{2}(.) \pm \sigma_{S} f_{3}(.)\right] \\
& -X_{1} e^{-r T_{1}}\left[N\left(a_{2}\right) \pm \sigma_{X_{1}} f_{1}(.) \pm \sigma_{X_{2}} f_{2}(.) \pm \sigma_{S} f_{3}(.)\right]
\end{aligned}
$$

For some $\sigma_{X_{i}}{ }^{\prime} s=1(i=1,2)$ with (i) All $\mu_{X_{1}}=\mu_{X_{2}}=\mu_{S}=1$ (ii) Some $\mu_{X_{i}}{ }^{\prime} s=1 \quad(i=1,2)$

And/or

$$
\begin{aligned}
C C_{\text {Stressed }}= & S e^{-q T_{2}}\left[N_{2}\left(a_{1}, b_{1} ; \sqrt{T_{1} / T_{2}}\right) \pm \sigma_{X_{1} X_{2} S} f_{1}(.) \pm \sigma_{X_{1} X_{2} S} f_{2}(.) \pm \sigma_{X_{1} X_{2} S} f_{3}(.)\right] \\
& -X_{2} e^{-r T_{2}}\left[N_{2}\left(a_{2}, b_{2} ; \sqrt{T_{1} / T_{2}}\right) \pm \sigma_{X_{1} X_{2} S} f_{1}(.) \pm \sigma_{X_{1} X_{2} S} f_{2}(.) \pm \sigma_{X_{1} X_{2} S} f_{3}(.)\right] \\
& -X_{1} e^{-r T_{1}}\left[N\left(a_{2}\right) \pm \sigma_{X_{1} X_{2} S} f_{1}(.) \pm \sigma_{X_{1} X_{2} S} f_{2}(.) \pm \sigma_{X_{1} X_{2} S} f_{3}(.)\right]
\end{aligned}
$$

For some $\sigma_{X_{i} X_{i} S}{ }^{\prime} s=1(i=1,2)$ with (i) All $\mu_{X_{1} X_{2} S}=1$ (ii) Some $\mu_{X_{1} X_{2} S}{ }^{\prime} s=1(i=1,2)$

\subsubsection{Proposed Jameel's CALL on PUT Stressed Option Models}

Class 1:

$$
\begin{aligned}
C P_{\text {Stressed }} & =X_{2} e^{-r T_{2}}\left[N_{2}\left(\mu_{X_{1}} \mu_{X_{2}} \mu_{S}\left(-a_{2},-b_{2} ; \sqrt{T_{1} / T_{2}}\right)\right) \pm \sigma_{X_{1}} f_{1}(.) \pm \sigma_{X_{2}} f_{2}(.) \pm \sigma_{S} f_{3}(.)\right] \\
& -S e^{-q T_{2}}\left[N_{2}\left(\mu_{X_{1}} \mu_{X_{2}} \mu_{S}\left(-a_{1},-b_{1} ; \sqrt{T_{1} / T_{2}}\right)\right) \pm \sigma_{X_{1}} f_{1}(.) \pm \sigma_{X_{2}} f_{2}(.) \pm \sigma_{S} f_{3}(.)\right] \\
& -X_{1} e^{-r T_{1}}\left[N\left(\mu_{X_{1}} \mu_{X_{2}} \mu_{S}\left(-a_{2}\right)\right) \pm \sigma_{X_{1}} f_{1}(.) \pm \sigma_{X_{2}} f_{2}(.) \pm \sigma_{S} f_{3}(.)\right]
\end{aligned}
$$

Class 2:

$$
\begin{aligned}
C P_{\text {Stressed }} & =X_{2} e^{-r T_{2}}\left[N_{2}\left(\mu_{X_{1} X_{2} S}\left(-a_{2},-b_{2} ; \sqrt{T_{1} / T_{2}}\right)\right) \pm \sigma_{X_{1} X_{2} S} f_{1}(.) \pm \sigma_{X_{1} X_{2} S} f_{2}(.) \pm \sigma_{X_{1} X_{2} S} f_{3}(.)\right] \\
& -S e^{-q T_{2}}\left[N_{2}\left(\mu_{X_{1} X_{2} S}\left(-a_{1},-b_{1} ; \sqrt{T_{1} / T_{2}}\right)\right) \pm \sigma_{X_{1} X_{2} S} f_{1}(.) \pm \sigma_{X_{1} X_{2} S} f_{2}(.) \pm \sigma_{X_{1} X_{2} S} f_{3}(.)\right] \\
& -X_{1} e^{-r T_{1}}\left[N\left(\mu_{X_{1} X_{2} S}\left(-a_{2}\right)\right) \pm \sigma_{X_{1} X_{2} S} f_{1}(.) \pm \sigma_{X_{1} X_{2} S} f_{2}(.) \pm \sigma_{X_{1} X_{2} S} f_{3}(.)\right]
\end{aligned}
$$

Where,

$$
\begin{aligned}
C P_{\text {Stressed }} & =X_{2} e^{-r T_{2}} N_{2}\left(-a_{2},-b_{2} ; \sqrt{T_{1} / T_{2}}\right) \\
& -S e^{-q T_{2}} N_{2}\left(-a_{1},-b_{1} ; \sqrt{T_{1} / T_{2}}\right) \\
& -X_{1} e^{-r T_{1}} N\left(-a_{2}\right)
\end{aligned}
$$


These are the general FORMS of the proposal, however, we can use COMBINATORICS to obtain different possible Combination of TERMS and SIGNS (to achieve different values of $C P_{\text {Stressed }}$ : positive, negative, high, low, close and so on).CLASS 1 and 2 are OPTIMUM at:

$$
\begin{aligned}
C P_{\text {Stressed }} & =X_{2} e^{-r T_{2}}\left[N_{2}\left(-a_{2},-b_{2} ; \sqrt{T_{1} / T_{2}}\right) \pm \sigma_{X_{1}} f_{1}(.) \pm \sigma_{X_{2}} f_{2}(.) \pm \sigma_{S} f_{3}(.)\right] \\
& -S e^{-q T_{2}}\left[N_{2}\left(-a_{1},-b_{1} ; \sqrt{T_{1} / T_{2}}\right) \pm \sigma_{X_{1}} f_{1}(.) \pm \sigma_{X_{2}} f_{2}(.) \pm \sigma_{S} f_{3}(.)\right] \\
& -X_{1} e^{-r T_{1}}\left[N\left(-a_{2}\right) \pm \sigma_{X_{1}} f_{1}(.) \pm \sigma_{X_{2}} f_{2}(.) \pm \sigma_{S} f_{3}(.)\right]
\end{aligned}
$$

For some $\sigma_{X_{i}}{ }^{\prime} s=1(i=1,2)$ with (i) All $\mu_{X_{1}}=\mu_{X_{2}}=\mu_{S}=1 \quad$ (ii) Some $\mu_{X_{i}}{ }^{\prime} s=1 \quad(i=1,2)$

And/or

$$
\begin{aligned}
C P_{\text {Stressed }} & =X_{2} e^{-r T_{2}}\left[N_{2}\left(-a_{2},-b_{2} ; \sqrt{T_{1} / T_{2}}\right) \pm \sigma_{X_{1} X_{2} S} f_{1}(.) \pm \sigma_{X_{1} X_{2} S} f_{2}(.) \pm \sigma_{X_{1} X_{2} S} f_{3}(.)\right] \\
& -S e^{-q T_{2}}\left[N_{2}\left(-a_{1},-b_{1} ; \sqrt{T_{1} / T_{2}}\right) \pm \sigma_{X_{1} X_{2} S} f_{1}(.) \pm \sigma_{X_{1} X_{2} S} f_{2}(.) \pm \sigma_{X_{1} X_{2} S} f_{3}(.)\right] \\
& -X_{1} e^{-r T_{1}}\left[N\left(-a_{2}\right) \pm \sigma_{X_{1} X_{2} S} f_{1}(.) \pm \sigma_{X_{1} X_{2} S} f_{2}(.) \pm \sigma_{X_{1} X_{2} S} f_{3}(.)\right]
\end{aligned}
$$

For some $\sigma_{X_{i} X_{i} S}{ }^{\prime} s=1(i=1,2)$ with (i) All $\mu_{X_{1} X_{2} S}=1$ (ii) Some $\mu_{X_{1} X_{2} S}{ }^{\prime} s=1(i=1,2)$

2.2.3 Proposed Jameel's PUT on CALL Stressed Option Models

Class 1:

$$
\begin{aligned}
P C_{\text {Stressed }} & =X_{2} e^{-r T_{2}}\left[N_{2}\left(\mu_{X_{1}} \mu_{X_{2}} \mu_{S}\left(-a_{2}, b_{2} ;-\sqrt{T_{1} / T_{2}}\right)\right) \pm \sigma_{X_{1}} f_{1}(.) \pm \sigma_{X_{2}} f_{2}(.) \pm \sigma_{S} f_{3}(.)\right] \\
& -S e^{-q T_{2}}\left[N_{2}\left(\mu_{X_{1}} \mu_{X_{2}} \mu_{S}\left(-a_{1},-b_{1} ;-\sqrt{T_{1} / T_{2}}\right)\right) \pm \sigma_{X_{1}} f_{1}(.) \pm \sigma_{X_{2}} f_{2}(.) \pm \sigma_{S} f_{3}(.)\right] \\
& +X_{1} e^{-r T_{1}}\left[N\left(\mu_{X_{1}} \mu_{X_{2}} \mu_{S}\left(-a_{2}\right)\right) \pm \sigma_{X_{1}} f_{1}(.) \pm \sigma_{X_{2}} f_{2}(.) \pm \sigma_{S} f_{3}(.)\right]
\end{aligned}
$$

Class 2:

$$
\begin{aligned}
P C_{\text {Stressed }} & =X_{2} e^{-r T_{2}}\left[N_{2}\left(\mu_{X_{1} X_{2} S}\left(-a_{2}, b_{2} ;-\sqrt{T_{1} / T_{2}}\right)\right) \pm \sigma_{X_{1} X_{2} S} f_{1}(.) \pm \sigma_{X_{1} X_{2} S} f_{2}(.) \pm \sigma_{X_{1} X_{2} S} f_{3}(.)\right] \\
& -S e^{-q T_{2}}\left[N_{2}\left(\mu_{X_{1} X_{2} S}\left(-a_{1},-b_{1} ;-\sqrt{T_{1} / T_{2}}\right)\right) \pm \sigma_{X_{1} X_{2} S} f_{1}(.) \pm \sigma_{X_{1} X_{2} S} f_{2}(.) \pm \sigma_{X_{1} X_{2} S} f_{3}(.)\right] \\
& +X_{1} e^{-r T_{1}}\left[N\left(\mu_{X_{1} X_{2} S}\left(-a_{2}\right)\right) \pm \sigma_{X_{1} X_{2} S} f_{1}(.) \pm \sigma_{X_{1} X_{2} S} f_{2}(.) \pm \sigma_{X_{1} X_{2} S} f_{3}(.)\right]
\end{aligned}
$$

Where,

$$
\begin{aligned}
P C=X_{2} e^{-r T_{2}}\left[N_{2}\left(-a_{2}, b_{2} ;-\sqrt{T_{1} / T_{2}}\right)\right] \\
-S e^{-q T_{2}}\left[N_{2}\left(-a_{1},-b_{1} ;-\sqrt{T_{1} / T_{2}}\right)\right] \\
+X_{1} e^{-r T_{1}}\left[N\left(-a_{2}\right)\right]
\end{aligned}
$$

These are the general FORMS of the proposal, however, we can use COMBINATORICS to obtain different possible Combination of TERMS and SIGNS (to achieve different values of $P C_{\text {Stressed }}$ : positive, negative, high, low, close and so on).CLASS 1 and 2 are OPTIMUM at: 


$$
\begin{aligned}
P C_{\text {Stressed }} & =X_{2} e^{-r T_{2}}\left[N_{2}\left(-a_{2}, b_{2} ;-\sqrt{T_{1} / T_{2}}\right) \pm \sigma_{X_{1}} f_{1}(.) \pm \sigma_{X_{2}} f_{2}(.) \pm \sigma_{S} f_{3}(.)\right] \\
& -S e^{-q T_{2}}\left[N_{2}\left(-a_{1},-b_{1} ;-\sqrt{T_{1} / T_{2}}\right) \pm \sigma_{X_{1}} f_{1}(.) \pm \sigma_{X_{2}} f_{2}(.) \pm \sigma_{S} f_{3}(.)\right] \\
& +X_{1} e^{-r T_{1}}\left[N\left(-a_{2}\right) \pm \sigma_{X_{1}} f_{1}(.) \pm \sigma_{X_{2}} f_{2}(.) \pm \sigma_{S} f_{3}(.)\right]
\end{aligned}
$$

For some $\sigma_{X_{i}}{ }^{\prime} s=1(i=1,2)$ with (i) All $\mu_{X_{1}}=\mu_{X_{2}}=\mu_{S}=1$ (ii) Some $\mu_{X_{i}}{ }^{\prime} s=1 \quad(i=1,2)$

And/or

$$
\begin{aligned}
P C_{\text {Stressed }} & =X_{2} e^{-r T_{2}}\left[N_{2}\left(-a_{2}, b_{2} ;-\sqrt{T_{1} / T_{2}}\right) \pm \sigma_{X_{1} X_{2} S} f_{1}(.) \pm \sigma_{X_{1} X_{2} S} f_{2}(.) \pm \sigma_{X_{1} X_{2} S} f_{3}(.)\right] \\
& -S e^{-q T_{2}}\left[N_{2}\left(-a_{1},-b_{1} ;-\sqrt{T_{1} / T_{2}}\right) \pm \sigma_{X_{1} X_{2} S} f_{1}(.) \pm \sigma_{X_{1} X_{2} S} f_{2}(.) \pm \sigma_{X_{1} X_{2} S} f_{3}(.)\right] \\
& +X_{1} e^{-r T_{1}}\left[N\left(-a_{2}\right) \pm \sigma_{X_{1} X_{2} S} f_{1}(.) \pm \sigma_{X_{1} X_{2} S} f_{2}(.) \pm \sigma_{X_{1} X_{2} S} f_{3}(.)\right]
\end{aligned}
$$

For some $\sigma_{X_{i} X_{i} S}{ }^{\prime} s=1(i=1,2)$ with (i) All $\mu_{X_{1} X_{2} S}=1$ (ii) Some $\mu_{X_{1} X_{2} S}{ }^{\prime} s=1(i=1,2)$

\subsubsection{Proposed Jameel's PUT on PUT Stressed Option Models}

\section{Class 1:}

$$
\begin{aligned}
P P_{\text {Stressed }}= & S e^{-q T_{2}}\left[N_{2}\left(\mu_{X_{1}} \mu_{X_{2}} \mu_{S}\left(a_{1},-b_{1} ;-\sqrt{T_{1} / T_{2}}\right)\right) \pm \sigma_{X_{1}} f_{1}(.) \pm \sigma_{X_{2}} f_{2}(.) \pm \sigma_{S} f_{3}(.)\right] \\
& -X_{2} e^{-r T_{2}}\left[N_{2}\left(\mu_{X_{1}} \mu_{X_{2}} \mu_{S}\left(a_{2},-b_{2} ;-\sqrt{T_{1} / T_{2}}\right)\right) \pm \sigma_{X_{1}} f_{1}(.) \pm \sigma_{X_{2}} f_{2}(.) \pm \sigma_{S} f_{3}(.)\right] \\
& +X_{1} e^{-r T_{1}}\left[N\left(\mu_{X_{1}} \mu_{X_{2}} \mu_{S}\left(a_{2}\right)\right) \pm \sigma_{X_{1}} f_{1}(.) \pm \sigma_{X_{2}} f_{2}(.) \pm \sigma_{S} f_{3}(.)\right]
\end{aligned}
$$

Class 2:

$$
\begin{aligned}
P P_{\text {Stressed }}= & S e^{-q T_{2}}\left[N_{2}\left(\mu_{X_{1} X_{2} S}\left(a_{1},-b_{1} ;-\sqrt{T_{1} / T_{2}}\right)\right) \pm \sigma_{X_{1} X_{2} S} f_{1}(.) \pm \sigma_{X_{1} X_{2} S} f_{2}(.) \pm \sigma_{X_{1} X_{2} S} f_{3}(.)\right] \\
& -X_{2} e^{-r T_{2}}\left[N_{2}\left(\mu_{X_{1} X_{2} S}\left(a_{2},-b_{2} ;-\sqrt{T_{1} / T_{2}}\right)\right) \pm \sigma_{X_{1} X_{2} S} f_{1}(.) \pm \sigma_{X_{1} X_{2} S} f_{2}(.) \pm \sigma_{X_{1} X_{2} S} f_{3}(.)\right] \\
& +X_{1} e^{-r T_{1}}\left[N\left(\mu_{X_{1} X_{2} S}\left(a_{2}\right)\right) \pm \sigma_{X_{1} X_{2} S} f_{1}(.) \pm \sigma_{X_{1} X_{2} S} f_{2}(.) \pm \sigma_{X_{1} X_{2} S} f_{3}(.)\right]
\end{aligned}
$$

Where,

$$
\begin{aligned}
P P=S e^{-q T_{2}} & {\left[N_{2}\left(a_{1},-b_{1} ;-\sqrt{T_{1} / T_{2}}\right)\right] } \\
& -X_{2} e^{-r T_{2}}\left[N_{2}\left(a_{2},-b_{2} ;-\sqrt{T_{1} / T_{2}}\right)\right] \\
& +X_{1} e^{-r T_{1}}\left[N\left(a_{2}\right)\right]
\end{aligned}
$$

These are the general FORMS of the proposal, however, we can use COMBINATORICS to obtain different possible Combination of TERMS and SIGNS (to achieve different values of $P P_{\text {Stressed }}$ : positive, negative, high, low, close and so on).CLASS 1 and 2 are OPTIMUM at:

$$
\begin{aligned}
P P_{\text {Stressed }}= & S e^{-q T_{2}}\left[N_{2}\left(a_{1},-b_{1} ;-\sqrt{T_{1} / T_{2}}\right) \pm \sigma_{X_{1}} f_{1}(.) \pm \sigma_{X_{2}} f_{2}(.) \pm \sigma_{S} f_{3}(.)\right] \\
& -X_{2} e^{-r T_{2}}\left[N_{2}\left(a_{2},-b_{2} ;-\sqrt{T_{1} / T_{2}}\right) \pm \sigma_{X_{1}} f_{1}(.) \pm \sigma_{X_{2}} f_{2}(.) \pm \sigma_{S} f_{3}(.)\right] \\
& +X_{1} e^{-r T_{1}}\left[N\left(a_{2}\right) \pm \sigma_{X_{1}} f_{1}(.) \pm \sigma_{X_{2}} f_{2}(.) \pm \sigma_{S} f_{3}(.)\right]
\end{aligned}
$$

For some $\sigma_{X_{i}}{ }^{\prime} s=1(i=1,2)$ with (i) All $\mu_{X_{1}}=\mu_{X_{2}}=\mu_{S}=1 \quad$ (ii) Some $\mu_{X_{i}}{ }^{\prime} s=1 \quad(i=1,2)$ 
And/or

$$
\begin{aligned}
P P_{\text {Stressed }}= & S e^{-q T_{2}}\left[N_{2}\left(a_{1},-b_{1} ;-\sqrt{T_{1} / T_{2}}\right) \pm \sigma_{X_{1} X_{2} S} f_{1}(.) \pm \sigma_{X_{1} X_{2} S} f_{2}(.) \pm \sigma_{X_{1} X_{2} S} f_{3}(.)\right] \\
& -X_{2} e^{-r T_{2}}\left[N_{2}\left(a_{2},-b_{2} ;-\sqrt{T_{1} / T_{2}}\right) \pm \sigma_{X_{1} X_{2} S} f_{1}(.) \pm \sigma_{X_{1} X_{2} S} f_{2}(.) \pm \sigma_{X_{1} X_{2} S} f_{3}(.)\right] \\
& +X_{1} e^{-r T_{1}}\left[N\left(a_{2}\right) \pm \sigma_{X_{1} X_{2} S} f_{1}(.) \pm \sigma_{X_{1} X_{2} S} f_{2}(.) \pm \sigma_{X_{1} X_{2} S} f_{3}(.)\right]
\end{aligned}
$$

For some $\sigma_{X_{i} X_{i} S}{ }^{\prime} s=1(i=1,2)$ with (i) All $\mu_{X_{1} X_{2} S}=1$ (ii) Some $\mu_{X_{1} X_{2} S}{ }^{\prime} s=1(i=1,2)$

Where, $N_{2}\left(a, b ; \sqrt{T_{1} / T_{2}}\right)$ is the cumulative distribution function of the BIVARIATE Normal Random Variable with Time $\sqrt{T_{1} / T_{2}}$, that is $N\left(a_{2}\right)=\frac{1}{\sigma \sqrt{2 \pi}} \int_{-\infty}^{a_{2}} e^{-\frac{\left(a_{2}-\mu\right)^{2}}{2 \sigma^{2}}} d a_{2}, N_{2}\left(a_{2}, b_{2} ; \sqrt{T_{1} / T_{2}}\right)=\frac{1}{2 \pi \sqrt{1-\left(\sqrt{T_{1} / T_{2}}\right)^{2}}} \int_{-\infty}^{a} \int_{-\infty}^{b} e^{-\frac{1}{2\left(1-\left(\sqrt{T_{1} / T_{2}}\right)^{2}\right)\left(a^{2}-2\left(\sqrt{T_{1} / T_{2}}\right) a b+b^{2}\right)}} d a d b$ $a_{1}=\frac{\log \left(S / X_{2}\right)+\left(r+\sigma^{2} / 2\right) T_{2}}{\sigma \sqrt{T_{2}}}, b_{1}=\frac{\log \left(S / X_{1}\right)+\left(r+\sigma^{2} / 2\right) T_{1}}{\sigma \sqrt{T_{1}}}, a_{2}=a_{1}-\sigma \sqrt{t_{n}}$ and $b_{2}=b_{1}-\sigma \sqrt{t_{1}}$.

\subsection{Proposed Jameel's Stressed Greeks}

\subsubsection{Proposed Jameel's Stressed DELTA Models}

\section{Class 1:}

$$
\begin{aligned}
\left(\frac{\partial V}{\partial S}\right)_{\text {Stressed }} & =\phi \omega r_{f} S e^{-r_{f} T_{2}}\left[N_{2}\left(-\mu_{X_{1}} \mu_{X_{2}} \mu_{S} \phi w\left(X_{2}-\sigma \sqrt{T_{1}}, \phi d_{+} ; \omega \sqrt{T_{1} / T_{2}}\right)\right) \pm \sigma_{X_{1}} f_{1}(.) \pm \sigma_{X_{2}} f_{2}(.) \pm \sigma_{S} f_{3}(.)\right] \\
& -\phi \omega r_{d} X_{1} e^{-r_{d} T_{2}}\left[N_{2}\left(-\mu_{X_{1}} \mu_{X_{2}} \mu_{S} \phi w\left(X_{2}, \phi d_{-} ; \omega \sqrt{T_{1} / T_{2}}\right)\right) \pm \sigma_{X_{1}} f_{1}(.) \pm \sigma_{X_{2}} f_{2}(.) \pm \sigma_{S} f_{3}(.)\right] \\
& -\omega r_{d} X_{1} e^{-r_{d} T_{1}}\left[N\left(-\mu_{X_{1}} \mu_{X_{2}} \mu_{S} \phi w X_{2}\right) \pm \sigma_{X_{1}} f_{1}(.) \pm \sigma_{X_{2}} f_{2}(.) \pm \sigma_{S} f_{3}(.)\right] \\
& -\frac{\sigma}{2} S e^{-r_{f} T_{2}}\left[\frac{1}{\sqrt{t}} n\left(X_{2}-\sigma \sqrt{T_{1}}\right)\left(N\left(\mu_{X_{1}} \mu_{X_{2}} \mu_{S} \phi d_{+}^{T_{2}}\right) \pm \sigma_{X_{1}} f_{1}(.) \pm \sigma_{X_{2}} f_{2}(.) \pm \sigma_{S} f_{3}(.)\right)\right] \\
& +\frac{\omega}{\sqrt{T_{2}}} n\left(d_{+}\right)\left[N\left(\mu_{X_{1}} \mu_{X_{2}} \mu_{S}\left(-\phi \omega \frac{X_{2} \sqrt{T_{2}}+\sqrt{T_{1}} d_{-}}{\sqrt{\tau}}\right)\right) \pm \sigma_{X_{1}} f_{1}(.) \pm \sigma_{X_{2}} f_{2}(.) \pm \sigma_{S} f_{3}(.)\right]
\end{aligned}
$$

\section{Class 2:}

$$
\begin{aligned}
\left(\frac{\partial V}{\partial S}\right)_{\text {Stressed }} & =\phi \omega r_{f} S e^{-r_{f} T_{2}}\left[N_{2}\left(-\mu_{X_{1} X_{2} S} \phi w\left(X_{2}-\sigma \sqrt{T_{1}}, \phi d_{+} ; \omega \sqrt{T_{1} / T_{2}}\right)\right) \pm \sigma_{X_{1} X_{2} s} f_{1}(.) \pm \sigma_{X_{1} X_{2} s} f_{2}(.) \pm \sigma_{X_{1} X_{2} s} f_{3}(.)\right] \\
& -\phi \omega r_{d} X_{1} e^{-r_{d} T_{2}}\left[N_{2}\left(-\mu_{X_{1} X_{2} S} \phi w\left(X_{2}, \phi d_{-} ; \omega \sqrt{T_{1} / T_{2}}\right)\right) \pm \sigma_{X_{1} X_{2} s} f_{1}(.) \pm \sigma_{X_{1} X_{2} s} f_{2}(.) \pm \sigma_{X_{1} X_{2} s} f_{3}(.)\right] \\
& -\omega r_{d} X_{1} e^{-r_{d} T_{1}}\left[N\left(-\mu_{X_{1} X_{2} S} \phi w X_{2}\right) \pm \sigma_{X_{1} X_{2} S} f_{1}(.) \pm \sigma_{X_{1} X_{2} S} f_{2}(.) \pm \sigma_{X_{1} X_{2} S} f_{3}(.)\right] \\
& -\frac{\sigma}{2} S e^{-r_{f} T_{2}}\left[\frac{1}{\sqrt{t}} n\left(X_{2}-\sigma \sqrt{T_{1}}\right)\left(N\left(\mu_{X_{1} X_{2} S} \phi d_{+}^{T_{2}}\right) \pm \sigma_{X_{1} X_{2} S} f_{1}(.) \pm \sigma_{X_{1} X_{2} S} f_{2}(.) \pm \sigma_{X_{1} X_{2} S} f_{3}(.)\right)\right] \\
& +\frac{\omega}{\sqrt{T_{2}}} n\left(d_{+}\right)\left[N\left(\mu_{X_{1} X_{2} s}\left(-\phi \omega \frac{X_{2} \sqrt{T_{2}}+\sqrt{T_{1}} d-}{\sqrt{\tau}}\right)\right) \pm \sigma_{X_{1} X_{2} S} f_{1}(.) \pm \sigma_{X_{1} X_{2} S} f_{2}(.) \pm \sigma_{X_{1} X_{2} S} f_{3}(.)\right]
\end{aligned}
$$

Where, 


$$
\begin{array}{rl}
\frac{\partial V}{\partial S}=\phi \omega r_{f} & S e^{-r_{f} T_{2}} N_{2}\left(-\phi w\left(X_{2}-\sigma \sqrt{T_{1}}, \phi d_{+} ; \omega \sqrt{T_{1} / T_{2}}\right)\right) \\
& -\phi \omega r_{d} X_{1} e^{-r_{d} T_{2}} N_{2}\left(-\phi w\left(X_{2}, \phi d_{-} ; \omega \sqrt{T_{1} / T_{2}}\right)\right) \\
& -\omega r_{d} X_{1} e^{-r_{d} T_{1}} N\left(-\phi w X_{2}\right) \\
& -\frac{\sigma}{2} S e^{-r_{f} T_{2}}\left[\frac{1}{\sqrt{t}} n\left(X_{2}-\sigma \sqrt{T_{1}}\right) N\left(\phi d_{+}^{T_{2}}\right)\right] \\
+ & \frac{\omega}{\sqrt{T_{2}}} n\left(d_{+}\right) N\left(-\phi \omega \frac{X_{2} \sqrt{T_{2}}+\sqrt{T_{1}} d d_{-}}{\sqrt{\tau}}\right)
\end{array}
$$

These are the general FORMS of the proposal, however, we can use COMBINATORICS to obtain different possible Combination of TERMS and SIGNS (to achieve different values of $\left(\frac{\partial V}{\partial S}\right)_{\text {Stressed }}$ : positive, negative, high, low, close and so on).CLASS 1 and 2 are OPTIMUM at:

$$
\begin{aligned}
\left(\frac{\partial V}{\partial S}\right)_{\text {stressed }} & =\phi \omega r_{f} S e^{-r_{f} T_{2}}\left[N_{2}\left(-\phi w\left(X_{2}-\sigma \sqrt{T_{1}}, \phi d_{+} ; \omega \sqrt{T_{1} / T_{2}}\right)\right) \pm \sigma_{X_{1}} f_{1}(.) \pm \sigma_{X_{2}} f_{2}(.) \pm \sigma_{S} f_{3}(.)\right] \\
& -\phi \omega r_{d} X_{1} e^{-r_{d} T_{2}}\left[N_{2}\left(-\phi w\left(X_{2}, \phi d_{-} ; \omega \sqrt{T_{1} / T_{2}}\right)\right) \pm \sigma_{X_{1}} f_{1}(.) \pm \sigma_{X_{2}} f_{2}(.) \pm \sigma_{S} f_{3}(.)\right] \\
& -\omega r_{d} X_{1} e^{-r_{d} T_{1}}\left[N\left(-\phi w X_{2}\right) \pm \sigma_{X_{1}} f_{1}(.) \pm \sigma_{X_{2}} f_{2}(.) \pm \sigma_{S} f_{3}(.)\right] \\
& -\frac{\sigma}{2} S e^{-r_{f} T_{2}}\left[\frac{1}{\sqrt{t}} n\left(X_{2}-\sigma \sqrt{T_{1}}\right)\left(N\left(\phi d_{+}^{T_{2}}\right) \pm \sigma_{X_{1}} f_{1}(.) \pm \sigma_{X_{2}} f_{2}(.) \pm \sigma_{S} f_{3}(.)\right)\right] \\
& +\frac{\omega}{\sqrt{T_{2}}} n\left(d_{+}\right)\left[N\left(\left(-\phi \omega \frac{X_{2} \sqrt{T_{2}}+\sqrt{T_{1}} d_{-}}{\sqrt{\tau}}\right)\right) \pm \sigma_{X_{1}} f_{1}(.) \pm \sigma_{X_{2}} f_{2}(.) \pm \sigma_{S} f_{3}(.)\right]
\end{aligned}
$$

For some $\sigma_{X_{i}}{ }^{\prime} s=1(i=1,2)$ with (i) All $\mu_{X_{1}}=\mu_{X_{2}}=\mu_{S}=1 \quad$ (ii) Some $\mu_{X_{i}}{ }^{\prime} s=1 \quad(i=1,2)$

And/or

$$
\begin{aligned}
\left(\frac{\partial V}{\partial S}\right)_{\text {Stressed }} & =\phi \omega r_{f} S e^{-r_{f} T_{2}}\left[N_{2}\left(-\phi w\left(X_{2}-\sigma \sqrt{T_{1}}, \phi d_{+} ; \omega \sqrt{T_{1} / T_{2}}\right)\right) \pm \sigma_{X_{1} X_{2} S} f_{1}(.) \pm \sigma_{X_{1} X_{2} S} f_{2}(.) \pm \sigma_{X_{1} X_{2} S} f_{3}(.)\right] \\
& -\phi \omega r_{d} X_{1} e^{-r_{d} T_{2}}\left[N_{2}\left(-\phi w\left(X_{2}, \phi d_{-} ; \omega \sqrt{T_{1} / T_{2}}\right)\right) \pm \sigma_{X_{1} X_{2} S} f_{1}(.) \pm \sigma_{X_{1} X_{2} S} f_{2}(.) \pm \sigma_{X_{1} X_{2} S} f_{3}(.)\right] \\
& -\omega r_{d} X_{1} e^{-r_{d} T_{1}}\left[N\left(-\phi w X_{2}\right) \pm \sigma_{X_{1} X_{2} S} f_{1}(.) \pm \sigma_{X_{1} X_{2} S} f_{2}(.) \pm \sigma_{X_{1} X_{2} S} f_{3}(.)\right] \\
& -\frac{\sigma}{2} S e^{-r_{f} T_{2}}\left[\frac{1}{\sqrt{t}} n\left(X_{2}-\sigma \sqrt{T_{1}}\right)\left(N\left(\phi d_{+}^{T_{2}}\right) \pm \sigma_{X_{1} X_{2} S} f_{1}(.) \pm \sigma_{X_{1} X_{2} S} f_{2}(.) \pm \sigma_{X_{1} X_{2} S} f_{3}(.)\right)\right] \\
& +\frac{\omega}{\sqrt{T_{2}}} n\left(d_{+}\right)\left[N\left(\left(-\phi \omega \frac{X_{2} \sqrt{T_{2}}+\sqrt{T_{1}} d_{-}}{\sqrt{\tau}}\right)\right) \pm \sigma_{X_{1} X_{2} S} f_{1}(.) \pm \sigma_{X_{1} X_{2} S} f_{2}(.) \pm \sigma_{X_{1} X_{2} S} f_{3}(.)\right]
\end{aligned}
$$

For some $\sigma_{X_{i} X_{i} S}{ }^{\prime} s=1(i=1,2)$ with (i) All $\mu_{X_{1} X_{2} S}=1$ (ii) Some $\mu_{X_{1} X_{2} S}{ }^{\prime} s=1(i=1,2)$

\subsubsection{ProposedJameel's Stressed GAMMA Models}

\section{Class 1:}

$$
\left(\frac{\partial^{2} V}{\partial S^{2}}\right)_{\text {Stressed }}=\phi \omega e^{-r_{f} T_{2}} \frac{\partial}{\partial S}\left[N_{2}\left(-\mu_{X_{1}} \mu_{X_{2}} \mu_{S} \phi w\left(X_{2}-\sigma \sqrt{T_{1}}, \phi d_{+} ; \omega \sqrt{T_{1} / T_{2}}\right)\right) \pm \sigma_{X_{1}} f_{1}(.) \pm \sigma_{X_{2}} f_{2}(.) \pm \sigma_{S} f_{3}(.)\right]
$$

\section{Class 2:}

$$
\left(\frac{\partial^{2} V}{\partial S^{2}}\right)_{\text {Stressed }}=\phi \omega e^{-r_{f} T_{2}} \frac{\partial}{\partial S}\left[N_{2}\left(-\mu_{X_{1} X_{2} S} \phi w\left(X_{2}-\sigma \sqrt{T_{1}}, \phi d_{+} ; \omega \sqrt{T_{1} / T_{2}}\right)\right) \pm \sigma_{X_{1} X_{2} S} f_{1}(.) \pm \sigma_{X_{1} X_{2} S} f_{2}(.) \pm \sigma_{X_{1} X_{2} S} f_{3}(.)\right]
$$


Where, $\frac{\partial^{2} V}{\partial S^{2}}=\phi \omega e^{-r_{f} T_{2}} \frac{\partial}{\partial S}\left[N_{2}\left(-\phi w\left(X_{2}-\sigma \sqrt{T_{1}}, \phi d_{+} ; \omega \sqrt{T_{1} / T_{2}}\right)\right)\right]$. These are the general FORMS of the proposal, however, we can use COMBINATORICS to obtain different possible Combination of TERMS and SIGNS (to achieve different values of $\left(\frac{\partial^{2} V}{\partial S^{2}}\right) \quad$ : positive, negative, high, low, close and so on).We can find the OPTIMUM values of GAMMA CLASS 1 and 2 in the same way as in the case of DELTA CLASS 1 and 2 above.

\subsubsection{Proposed Jameel's Stressed THETA Models}

Class 1:

$$
\begin{aligned}
\left(\frac{\partial V}{\partial T}\right)_{\text {Srressed }} & =\phi \omega r_{f} S e^{-r_{f} T_{2}}\left[N_{2}\left(-\mu_{X_{1}} \mu_{X_{2}} \mu_{S} \phi w\left(X_{2}-\sigma \sqrt{T_{1}}, \phi d_{+} ; \omega \sqrt{T_{1} / T_{2}}\right)\right) \pm \sigma_{X_{1}} f_{1}(.) \pm \sigma_{X_{2}} f_{2}(.) \pm \sigma_{S} f_{3}(.)\right] \\
& -\phi \omega r_{d} X_{1} e^{-r_{d} T_{2}}\left[N_{2}\left(-\mu_{X_{1}} \mu_{X_{2}} \mu_{S} \phi w\left(X_{2}, \phi d_{-} ; \omega \sqrt{T_{1} / T_{2}}\right)\right) \pm \sigma_{X_{1}} f_{1}(.) \pm \sigma_{X_{2}} f_{2}(.) \pm \sigma_{S} f_{3}(.)\right] \\
& -\omega r_{d} X_{1} e^{-r_{d} T_{1}}\left[N\left(-\mu_{X_{1}} \mu_{X_{2}} \mu_{S} \phi w X_{2}\right) \pm \sigma_{X_{1}} f_{1}(.) \pm \sigma_{X_{2}} f_{2}(.) \pm \sigma_{S} f_{3}(.)\right] \\
& -\frac{\sigma}{2} S e^{-r_{f} T_{2}}\left[\begin{array}{l}
\frac{1}{\sqrt{t}} n\left(X_{2}-\sigma \sqrt{T_{1}}\right)\left(N\left(\mu_{X_{1}} \mu_{X_{2}} \mu_{S} \phi d_{+}^{\tau}\right) \pm \sigma_{X_{1}} f_{1}(.) \pm \sigma_{X_{2}} f_{2}(.) \pm \sigma_{S} f_{3}(.)\right) \\
+\frac{\omega}{\sqrt{T_{2}}} n\left(d_{+}\right)\left(N\left(\mu_{X_{1}} \mu_{X_{2}} \mu_{S}\left(-\phi \omega \frac{X_{2} \sqrt{T_{2}}+\sqrt{T_{1}} d}{\sqrt{\tau}}\right)\right) \pm \sigma_{X_{1}} f_{1}(.) \pm \sigma_{X_{2}} f_{2}(.) \pm \sigma_{S} f_{3}(.)\right)
\end{array}\right)
\end{aligned}
$$

\section{Class 2:}

$$
\begin{aligned}
& \left(\frac{\partial V}{\partial T}\right)_{\text {stressed }}=\phi \omega r_{f} S e^{-r_{f} T_{2}}\left[N_{2}\left(-\mu_{X_{1} X_{2} S} \phi w\left(X_{2}-\sigma \sqrt{T_{1}}, \phi d_{+} ; \omega \sqrt{T_{1} / T_{2}}\right)\right) \pm \sigma_{X_{1} X_{2} S} f_{1}(.) \pm \sigma_{X_{1} X_{2} S} f_{2}(.) \pm \sigma_{X_{1} X_{2} S} f_{3}(.)\right] \\
& -\phi \omega r_{d} X_{1} e^{-r_{d} T_{2}}\left[N_{2}\left(-\mu_{X_{1} X_{2} s} \phi w\left(X_{2}, \phi d_{-} ; \omega \sqrt{T_{1} / T_{2}}\right)\right) \pm \sigma_{X_{1} X_{2} S} f_{1}(.) \pm \sigma_{X_{1} X_{2} s} f_{2}(.) \pm \sigma_{X_{1} X_{2} S} f_{3}(.)\right] \\
& -\omega r_{d} X_{1} e^{-r_{d} T_{1}}\left[N\left(-\mu_{X_{1} X_{2} s} \phi w X_{2}\right) \pm \sigma_{X_{1} X_{2} s} f_{1}(.) \pm \sigma_{X_{1} X_{2} s} f_{2}(.) \pm \sigma_{X_{1} X_{2} s} f_{3}(.)\right] \\
& -\frac{\sigma}{2} S e^{-r_{f} T_{2}}\left[\begin{array}{l}
\frac{1}{\sqrt{t}} n\left(X_{2}-\sigma \sqrt{T_{1}}\right)\left(N\left(\mu_{X_{1} X_{2} s} \phi d_{+}^{\tau}\right) \pm \sigma_{X_{1} X_{2} S} f_{1}(.) \pm \sigma_{X_{1} X_{2} S} f_{2}(.) \pm \sigma_{X_{1} X_{2} s} f_{3}(.)\right) \\
+\frac{\omega}{\sqrt{T_{2}}} n\left(d_{+}\right)\left(N\left(\mu_{X_{1} X_{2} s}\left(-\phi \omega \frac{X_{2} \sqrt{T_{2}}+\sqrt{T_{1}} d_{-}}{\sqrt{\tau}}\right)\right) \pm \sigma_{X_{1} X_{2} S} f_{1}(.) \pm \sigma_{X_{1} X_{2} S} f_{2}(.) \pm \sigma_{X_{1} X_{2} S} f_{3}(.)\right)
\end{array}\right]
\end{aligned}
$$

Where,

$$
\begin{array}{rl}
\frac{\partial V}{\partial T}=\phi \omega r_{f} & S e^{-r_{f} T_{2}} N_{2}\left(-\phi w\left(X_{2}-\sigma \sqrt{T_{1}}, \phi d_{+} ; \omega \sqrt{T_{1} / T_{2}}\right)\right) \\
& -\phi \omega r_{d} X_{1} e^{-r_{d} T_{2}} N_{2}\left(-\phi w\left(X_{2}, \phi d_{-} ; \omega \sqrt{T_{1} / T_{2}}\right)\right) \\
& -\omega r_{d} X_{1} e^{-r_{d} T_{1}} N\left(-\phi w X_{2}\right) \\
& -\frac{\sigma}{2} S e^{-r_{f} T_{2}}\left[\frac{1}{\sqrt{t}} n\left(X_{2}-\sigma \sqrt{T_{1}}\right) N\left(\phi d_{+}^{\tau}\right)+\frac{\omega}{\sqrt{T_{2}}} n\left(d_{+}\right) N\left(-\phi \omega \frac{X_{2} \sqrt{T_{2}}+\sqrt{T_{1}} d_{-}}{\sqrt{\tau}}\right)\right]
\end{array}
$$

These are the general FORMS of the proposal, however, we can use COMBINATORICS to obtain different possible Combination of TERMS and SIGNS (to achieve different values of $\left(\frac{\partial V}{\partial T}\right)_{\text {stressed }}$ : positive, negative, high, low, close and so on).We can find the OPTIMUM values of THETA CLASS 1 and 2 in the same way as in the case of DELTA CLASS 1 and 2 above.

\subsubsection{Proposed Jameel's Stressed RHO Models}

\section{Class 1:}

$$
\left(\frac{\partial V}{\partial r_{f}}\right)_{\text {Stressed }}=-\phi \omega T_{2} S e^{-r_{f} T_{2}}\left[N_{2}\left(-\mu_{X_{1}} \mu_{X_{2}} \mu_{S} \phi \omega\left(X_{2}-\sigma \sqrt{T_{1}}, \phi d_{+} ; \omega \sqrt{T_{1} / T_{2}}\right)\right) \pm \sigma_{X_{1}} f_{1}(.) \pm \sigma_{X_{2}} f_{2}(.) \pm \sigma_{S} f_{3}(.)\right]
$$




\section{Class 2:}

$$
\left(\frac{\partial V}{\partial r_{f}}\right)_{\text {Stressed }}=-\phi \omega T_{2} S e^{-r_{f} T_{2}}\left[N_{2}\left(-\mu_{X_{1} X_{2} S} \phi \omega\left(X_{2}-\sigma \sqrt{T_{1}}, \phi d_{+} ; \omega \sqrt{T_{1} / T_{2}}\right)\right) \pm \sigma_{X_{1} X_{2} S} f_{1}(.) \pm \sigma_{X_{1} X_{2} S} f_{2}(.) \pm \sigma_{X_{1} X_{2} S} f_{3}(.)\right]
$$

Where, $\frac{\partial V}{\partial r_{f}}=-\phi \omega T_{2} S e^{-r_{f} T_{2}} N_{2}\left(-\phi \omega\left(X_{2}-\sigma \sqrt{T_{1}}, \phi d_{+} ; \omega \sqrt{T_{1} / T_{2}}\right)\right)$.

These are the general FORMS of the proposal, however, we can use COMBINATORICS to obtain different possible Combination of TERMS and SIGNS (to achieve different values of $\left(\frac{\partial V}{\partial r_{f}}\right)_{\text {stressed }}$ : positive, negative, high, low, close and so on).We can find the OPTIMUM values of RHO CLASS 1 and 2 in the same way as in the case of DELTA CLASS 1 and 2 above.

\subsubsection{Proposed Jameel's Stressed VEGA Models}

\section{Class 1:}

$$
\left(\frac{\partial V}{\partial \sigma}\right)_{\text {Stressed }}=S e^{-r_{f} T_{2}}\left[\begin{array}{l}
\sqrt{t} n\left(X_{2}-\sigma \sqrt{T_{1}}\right)\left(N\left(\mu_{X_{1}} \mu_{X_{2}} \mu_{S} \phi d_{+}^{\tau}\right) \pm \sigma_{X_{1}} f_{1}(.) \pm \sigma_{X_{2}} f_{2}(.) \pm \sigma_{S} f_{3}(.)\right) \\
+\omega \sqrt{T_{2}} n\left(d_{+}\right)\left(N\left(\mu_{X_{1}} \mu_{X_{2}} \mu_{S}\left(-\phi \omega \frac{X_{2} \sqrt{T_{2}}+\sqrt{T_{1}} d_{-}}{\sqrt{\tau}}\right)\right) \pm \sigma_{X_{1}} f_{1}(.) \pm \sigma_{X_{2}} f_{2}(.) \pm \sigma_{S} f_{3}(.)\right)
\end{array}\right]
$$

\section{Class 2:}

$$
\begin{aligned}
& \left(\frac{\partial V}{\partial \sigma}\right)_{\text {Stressed }}=S e^{-r_{f} T_{2}}\left[\begin{array}{l}
\sqrt{t} n\left(X_{2}-\sigma \sqrt{T_{1}}\right)\left(N\left(\mu_{X_{1} X_{2} S} \phi d_{+}^{\tau}\right) \pm \sigma_{X_{1} X_{2} S} f_{1}(.) \pm \sigma_{X_{1} X_{2} S} f_{2}(.) \pm \sigma_{X_{1} X_{2} S} f_{3}(.)\right) \\
+\omega \sqrt{T_{2}} n\left(d_{+}\right)\left(N\left(\mu_{X_{1} X_{2} S}\left(-\phi \omega \frac{X_{2} \sqrt{T_{2}}+\sqrt{T_{1}} d_{-}}{\sqrt{\tau}}\right)\right) \pm \sigma_{X_{1} X_{2} S} f_{1}(.) \pm \sigma_{X_{1} X_{2} S} f_{2}(.) \pm \sigma_{X_{1} X_{2} S} f_{3}(.)\right)
\end{array}\right) \\
& \frac{\partial V}{\partial \sigma}=S e^{-r_{f} T_{2}}\left[\sqrt{t} n\left(X_{2}-\sigma \sqrt{T_{1}}\right) N\left(\phi d_{+}^{\tau}\right)+\omega \sqrt{T_{2}} n\left(d_{+}\right) N\left(-\phi \omega \frac{\left.X_{2} \sqrt{T_{2}}+\sqrt{T_{1}} d_{-}\right)}{\sqrt{\tau}}\right)\right] .
\end{aligned}
$$

These are the general FORMS of the proposal, however, we can use COMBINATORICS to obtain different possible Combination of TERMS and SIGNS (to achieve different values of $\left(\frac{\partial V}{\partial \sigma}\right)_{\text {Stressed }}$ : positive, negative, high, low, close and so on).We can find the OPTIMUM values of VEGA CLASS 1 and 2 in the same way as in the case of DELTA CLASS 1 and 2 above.

2.4 Proposed Jameel's Advanced Stressed Exotic Options Pricing Models

\subsubsection{Simple Chooser Options}

\section{Class 1:}

$$
\begin{aligned}
& \text { Simple }^{\text {Chooser }} \text { Stressed }=S e^{-D T}\left[N\left(\mu_{S} \mu_{K} d_{1}\right) \pm \sigma_{S} f_{1}(.) \pm \sigma_{K} f_{2}(.)\right]-K e^{-r T}\left[N\left(\mu_{S} \mu_{K} d_{2} \pm \sigma_{S} f_{1}(.) \pm \sigma_{K} f_{2}(.)\right)\right] \\
&-S e^{-D T}\left[N\left(-\mu_{S} \mu_{K} e_{1}\right) \pm \sigma_{S} f_{1}(.) \pm \sigma_{K} f_{2}(.)\right]+K e^{-r T}\left[N\left(-\mu_{S} \mu_{K} e_{2}\right) \pm \sigma_{S} f_{1}(.) \pm \sigma_{K} f_{2}(.)\right]
\end{aligned}
$$

Class 2:

$$
\begin{aligned}
& \text { simple Chooser }_{\text {Stressed }}=S e^{-D T}\left[N\left(\mu_{S K} d_{1}\right) \pm \sigma_{S K} f_{1}(.) \pm \sigma_{S K} f_{2}(.)\right]-K e^{-r T}\left[N\left(\mu_{S K} d_{2} \pm \sigma_{S K} f_{1}(.) \pm \sigma_{S K} f_{2}(.)\right)\right] \\
&-S e^{-D T}\left[N\left(-\mu_{S K} e_{1}\right) \pm \sigma_{S K} f_{1}(.) \pm \sigma_{S K} f_{2}(.)\right]+K e^{-r T}\left[N\left(-\mu_{S K} e_{2}\right) \pm \sigma_{S K} f_{1}(.) \pm \sigma_{S K} f_{2}(.)\right]
\end{aligned}
$$

Where, Simple Chooser $=S e^{-D T} N\left(d_{1}\right)-K e^{-r T} N\left(d_{2}\right)-S e^{-D T} N\left(-e_{1}\right)+K e^{-r T} N\left(-e_{2}\right)$ 
$d_{1}=\frac{\ln (S / K)+\left(r-D+0.5 \sigma^{2}\right) T}{\sigma \sqrt{T}}, e_{1}=\frac{\ln (S / K)+(r-D)+0.5 \sigma^{2} t}{\sigma \sqrt{t}}, d_{2}=d_{1}-\sigma \sqrt{T}$

And $e_{2}=e_{1}-\sigma \sqrt{T}$. These are the general FORMS of the proposal, however, we can use COMBINATORICS to obtain different possible Combination of TERMS and SIGNS (to achieve different values of ${ }^{\text {simple }}$ Chooser $_{\text {Stressed }}$ : positive, negative, high, low, close and so on).We can find the OPTIMUM values of SIMPLE CHOOSER OPTIONS CLASS 1 and 2 in the same way as in the case of COMPOUND OPTIONS CLASS 1 and 2 above.

\subsubsection{Complex Chooser Options}

Class 1:

$$
\begin{aligned}
\text { complex Chooser }_{\text {Stressed }} & =S e^{-d T_{1}}\left[N_{2}\left(\mu_{S} \mu_{K_{1}} \mu_{K_{2}}\left(x, y_{1} ; \rho_{1}\right)\right) \pm \sigma_{K_{1}} f_{1}(.) \pm \sigma_{K_{2}} f_{2}(.) \pm \sigma_{S} f_{3}(.)\right] \\
& -K_{1} e^{-r T_{1}}\left[N_{2}\left(\mu_{S} \mu_{K_{1}} \mu_{K_{2}}\left(x-\sigma \sqrt{T_{1}}, y_{1}-\sigma \sqrt{T_{1}} ; \rho_{1}\right)\right) \pm \sigma_{K_{1}} f_{1}(.) \pm \sigma_{K_{2}} f_{2}(.) \pm \sigma_{S} f_{3}(.)\right] \\
& -S e^{-d T_{2}}\left[N_{2}\left(\mu_{S} \mu_{K_{1}} \mu_{K_{2}}\left(-x,-y_{2} ; \rho_{2}\right)\right) \pm \sigma_{K_{1}} f_{1}(.) \pm \sigma_{K_{2}} f_{2}(.) \pm \sigma_{S} f_{3}(.)\right] \\
& +K_{2} e^{-r T_{2}}\left[N_{2}\left(\mu_{S} \mu_{K_{1}} \mu_{K_{2}}\left(-x+\sigma \sqrt{T_{2}},-y_{2}+\sigma \sqrt{T_{2}} ; \rho_{2}\right)\right) \pm \sigma_{K_{1}} f_{1}(.) \pm \sigma_{K_{2}} f_{2}(.) \pm \sigma_{S} f_{3}(.)\right]
\end{aligned}
$$

\section{Class 2:}

$$
\begin{aligned}
{ }^{\text {Complex }} \text { Chooser } & =S e^{-d T_{1}}\left[N_{2}\left(\mu_{S K_{1} K_{2}}\left(x, y_{1} ; \rho_{1}\right)\right) \pm \sigma_{S K_{1} K_{2}} f_{1}(.) \pm \sigma_{S K_{1} K_{2}} f_{2}(.) \pm \sigma_{S K_{1} K_{2}} f_{3}(.)\right] \\
& -K_{1} e^{-r T_{1}}\left[N_{2}\left(\mu_{S K_{1} K_{2}}\left(x-\sigma \sqrt{T_{1}}, y_{1}-\sigma \sqrt{T_{1}} ; \rho_{1}\right)\right) \pm \sigma_{S K_{1} K_{2}} f_{1}(.) \pm \sigma_{S K_{1} K_{2}} f_{2}(.) \pm \sigma_{S K_{1} K_{2}} f_{3}(.)\right] \\
& -S e^{-d T_{2}}\left[N_{2}\left(\mu_{S K_{1} K_{2}}\left(-x,-y_{2} ; \rho_{2}\right)\right) \pm \sigma_{S K_{1} K_{2}} f_{1}(.) \pm \sigma_{S K_{1} K_{2}} f_{2}(.) \pm \sigma_{S K_{1} K_{2}} f_{3}(.)\right] \\
& +K_{2} e^{-r T_{2}}\left[N_{2}\left(\mu_{S K_{1} K_{2}}\left(-x+\sigma \sqrt{T_{2}},-y_{2}+\sigma \sqrt{T_{2}} ; \rho_{2}\right)\right) \pm \sigma_{S K_{1} K_{2}} f_{1}(.) \pm \sigma_{S K_{1} K_{2}} f_{2}(.) \pm \sigma_{S K_{1} K_{2}} f_{3}(.)\right]
\end{aligned}
$$

$$
\begin{aligned}
& \text { Where, complex Chooser }=S e^{-d T_{1}} N_{2}\left(x, y_{1} ; \rho_{1}\right)-K_{1} e^{-r T_{1}} N_{2}\left(x-\sigma \sqrt{T_{1}}, y_{1}-\sigma \sqrt{T_{1}} ; \rho_{1}\right) \\
&-S e^{-d T_{2}} N_{2}\left(-x,-y_{2} ; \rho_{2}\right)+K_{2} e^{-r T_{2}} N_{2}\left(-x+\sigma \sqrt{T_{2}},-y_{2}+\sigma \sqrt{T_{2}} ; \rho_{2}\right) \\
& x=\frac{\ln \left(S e^{-d \tau} / X e^{-r \tau}\right)}{\sigma \sqrt{\tau}}+\frac{1}{2} \sigma \sqrt{\tau}, \quad y_{i}=\frac{\ln \left(S e^{-d T_{i}} / K_{i} e^{-r T_{i}}\right)}{\sigma \sqrt{T_{i}}}+\frac{1}{2} \sigma \sqrt{T_{i}} \text { and } \rho_{i}=\sqrt{\frac{\tau}{T_{i}}} .
\end{aligned}
$$

These are the general FORMS of the proposal, however, we can use COMBINATORICS to obtain different possible Combination of TERMS and SIGNS (to achieve different values of ${ }^{\text {Complex }}$ Chooser $_{\text {Stressed }}$ : positive, negative, high, low, close and so on).We can find the OPTIMUM values of COMPLEX CHOOSER OPTIONS CLASS 1 and 2 in the same way as in the case of COMPOUND OPTIONS CLASS 1 and 2 above.

\subsubsection{Lookback Options}

The Stressed FixedLookback Options Call Options Pricing Models are given by:

\section{Class 1:}

$$
\begin{gathered}
C_{L B(\text { Stressed })}=S e^{-d T}\left[N\left(\mu_{S} \mu_{M}(x+\sigma \sqrt{T})\right) \pm \sigma_{S} f_{1}(.) \pm \sigma_{M} f_{2}(.)\right]-M e^{-r T}\left[N\left(\mu_{S} \mu_{M}(x)\right) \pm \sigma_{S} f_{1}(.) \pm \sigma_{M} f_{2}(.)\right] \\
\quad+\frac{S}{B}\left(e^{-r T}(S / M)^{-B}\left[N\left(\mu_{S} \mu_{M}(y+B \sigma \sqrt{T})\right) \pm \sigma_{S} f_{1}(.) \pm \sigma_{M} f_{2}(.)\right]-e^{-d T}\left[N\left(\mu_{S} \mu_{M}(y)\right) \pm \sigma_{S} f_{1}(.) \pm \sigma_{M} f_{2}(.)\right]\right)
\end{gathered}
$$

\section{Class 2:}

$$
\begin{aligned}
& C_{L B(\text { Stressed })}=S e^{-d T}\left[N\left(\mu_{S M}(x+\sigma \sqrt{T})\right) \pm \sigma_{S M} f_{1}(.) \pm \sigma_{S M} f_{2}(.)\right]-M e^{-r T}\left[N\left(\mu_{S M}(x)\right) \pm \sigma_{S M} f_{1}(.) \pm \sigma_{S M} f_{2}(.)\right] \\
& \quad+\frac{S}{B}\left(e^{-r T}(S / M)^{-B}\left[N\left(\mu_{S M}(y+B \sigma \sqrt{T})\right) \pm \sigma_{S M} f_{1}(.) \pm \sigma_{S M} f_{2}(.)\right]-e^{-d T}\left[N\left(\mu_{S M}(y)\right) \pm \sigma_{S M} f_{1}(.) \pm \sigma_{S M} f_{2}(.)\right]\right)
\end{aligned}
$$


The Stressed FixedLookback Options Put Options Pricing Models are given by:

\section{Class 1:}

$$
\begin{aligned}
P_{L B(\text { Stressed })} & =-S e^{-d T}\left[N\left(\mu_{S} \mu_{M}(-x-\sigma \sqrt{T})\right) \pm \sigma_{S} f_{1}(.) \pm \sigma_{M} f_{2}(.)\right]+M e^{-r T}\left[N\left(\mu_{S} \mu_{M}(-x)\right) \pm \sigma_{S} f_{1}(.) \pm \sigma_{M} f_{2}(.)\right] \\
& -\frac{S}{B}\left(e^{-r T}(S / M)^{-B}\left[N\left(\mu_{S} \mu_{M}(-y-B \sigma \sqrt{T})\right) \pm \sigma_{S} f_{1}(.) \pm \sigma_{M} f_{2}(.)\right]-e^{-d T}\left[N\left(\mu_{S} \mu_{M}(-y)\right) \pm \sigma_{S} f_{1}(.) \pm \sigma_{M} f_{2}(.)\right]\right)
\end{aligned}
$$

\section{Class 2:}

$$
\begin{aligned}
P_{L B(\text { Stressed })} & =-S e^{-d T}\left[N\left(\mu_{S M}(-x-\sigma \sqrt{T})\right) \pm \sigma_{S M} f_{1}(.) \pm \sigma_{S M} f_{2}(.)\right]+M e^{-r T}\left[N\left(\mu_{S M}(-x)\right) \pm \sigma_{S M} f_{1}(.) \pm \sigma_{S M} f_{2}(.)\right] \\
& -\frac{S}{B}\left(e^{-r T}(S / M)^{-B}\left[N\left(\mu_{S M}(-y-B \sigma \sqrt{T})\right) \pm \sigma_{S M} f_{1}(.) \pm \sigma_{S M} f_{2}(.)\right]-e^{-d T}\left[N\left(\mu_{S M}(-y)\right) \pm \sigma_{S M} f_{1}(.) \pm \sigma_{S M} f_{2}(.)\right]\right)
\end{aligned}
$$

Where, the Fixed Lookback Call and Fixed Lookback Put are respectively given by:

$$
C_{L B}=S e^{-d T} N(x+\sigma \sqrt{T})-M e^{-r T} N(x)+\frac{S}{B}\left(e^{-r T}(S / M)^{-B} N(y+B \sigma \sqrt{T})-e^{-d T} N(y)\right)
$$

And $P_{L B}=-S e^{-d T} N(-x-\sigma \sqrt{T})+M e^{-r T} N(-x)-\frac{S}{B}\left(e^{-r T}(S / M)^{-B} N(-y-B \sigma \sqrt{T})-e^{-d T} N(-y)\right)$

$$
B=\frac{2(r-d)}{\sigma^{2}}, \quad x=\frac{\ln (S / M)+\left((r-d)-\frac{1}{2} \sigma^{2}\right) T}{\sigma \sqrt{T}} \text { and } y=\frac{-\ln (S / M)-\left((r-d)+\frac{1}{2} \sigma^{2}\right) T}{\sigma \sqrt{T}} \text {. }
$$

These are the general FORMS of the proposal, however, we can use COMBINATORICS to obtain different possible Combination of TERMS and SIGNS (to achieve different values of $C_{L B(\text { Stressed })}$ and $P_{L B(\text { Stressed })}$ : positive, negative, high, low, close and so on).We can find the OPTIMUM values of FIXEDLOOKBACK OPTIONS CALL AND FIXEDLOOKBACK OPTIONS PUT CLASS 1 and 2 in the same way as in the case of COMPOUND OPTIONS CLASS 1 and 2 above.

2.4.4 Barrier Options

The Stressed Down - and - in Call Barrier Options Pricing Models are given by:

\section{Class 1:}

$$
\begin{aligned}
{ }^{\text {Down-and-In }} C_{\text {Stressed }} & =S e^{-q t}(B / S)^{2 \lambda}\left[N\left(\mu_{S} \mu_{K} y\right) \pm \sigma_{S} f_{1}(.) \pm \sigma_{K} f_{2}(.)\right] \\
& -K e^{-r t}(B / S)^{2 \lambda-2}\left[N\left(\mu_{S} \mu_{K}(y-\sigma \sqrt{T})\right) \pm \sigma_{S} f_{1}(.) \pm \sigma_{K} f_{2}(.)\right]
\end{aligned}
$$

\section{Class 2:}

$$
\begin{aligned}
{ }^{\text {Down-and-In }} C_{\text {Stressed }} & =S e^{-q t}(B / S)^{2 \lambda}\left[N\left(\mu_{S K} y\right) \pm \sigma_{S K} f_{1}(.) \pm \sigma_{S K} f_{2}(.)\right] \\
& -K e^{-r t}(B / S)^{2 \lambda-2}\left[N\left(\mu_{S K}(y-\sigma \sqrt{T})\right) \pm \sigma_{S K} f_{1}(.) \pm \sigma_{S K} f_{2}(.)\right]
\end{aligned}
$$

Where,

$$
{ }_{\text {Down-and-In }} C=S e^{-q t}(B / S)^{2 \lambda} N(y)-K e^{-r t}(B / S)^{2 \lambda-2} N(y-\sigma \sqrt{T}) \quad, \quad \lambda=\frac{r-q+r^{2} / 2}{\sigma^{2}} \quad \text { and }
$$

$y=\frac{\ln \left[B^{2} /(S K)\right]}{\sigma \sqrt{T}}+\lambda \sigma \sqrt{T}$. B is the barrier level and assumed to be lower than the initial stock price.These are the general FORMS of the proposal, however, we can use COMBINATORICS to obtain different possible Combination of TERMS and SIGNS (to achieve different values of Down-and-In $^{\text {Stressed }}$ : positive, negative, high, low, close and so 
on).We can find the OPTIMUM values of DOWN - AND - IN CALL BARRIER OPTIONS CLASS 1 and 2 in the same way as in the case of COMPOUND OPTIONS CLASS 1 and 2 above.

2.4.5 Cash or Nothing Options:

Class 1:

$$
C N_{\text {Stressed }}=X e^{-r T}\left[N\left(\mu_{X}(\phi x-\phi \sigma \sqrt{T})\right) \pm \sigma_{X} f_{1}(.)\right]
$$

Class 2:

$$
C N_{\text {Stressed }}=X e^{-r T}\left[N\left(\mu_{X}(\phi x-\phi \sigma \sqrt{T})\right) \pm \sigma_{X} f_{1}(.)\right]
$$

Where, $C N=X e^{-r T} N(\phi x-\phi \sigma \sqrt{T})$

These are the general FORMS of the proposal, however, we can use COMBINATORICS to obtain different possible Combination of TERMS and SIGNS (to achieve different values of $C N_{\text {Stressed }}$ : positive, negative, high, low, close and so on).We can find the OPTIMUM values of CASH OR NOTHING OPTIONS CLASS 1 and 2 in the same way as in the case of COMPOUND OPTIONS CLASS 1 and 2 above.

\subsubsection{Gap Options}

Class 1:

$$
\begin{aligned}
G a p_{\text {stressed }} & =\phi S e^{-d T}\left[N\left(\mu_{S} \mu_{X} \phi x\right) \pm \sigma_{S} f_{1}(.) \pm \sigma_{X} f_{2}(.)\right] \\
& -\phi X e^{-r T}\left[N\left(\mu_{S} \mu_{X}(\phi x-\phi \sigma \sqrt{T})\right) \pm \sigma_{S} f_{1}(.) \pm \sigma_{X} f_{2}(.)\right]
\end{aligned}
$$

\section{Class 2:}

$$
\begin{aligned}
\text { Gap } p_{\text {Stressed }} & =\phi S e^{-d T}\left[N\left(\mu_{S X} \phi x\right) \pm \sigma_{S X} f_{1}(.) \pm \sigma_{S X} f_{2}(.)\right] \\
& -\phi X e^{-r T}\left[N\left(\mu_{S X}(\phi x-\phi \sigma \sqrt{T})\right) \pm \sigma_{S X} f_{1}(.) \pm \sigma_{S X} f_{2}(.)\right]
\end{aligned}
$$

Where, $G$ ap $=\phi S e^{-d T} N(\phi x)-\phi X e^{-r T} N(\phi x-\phi \sigma \sqrt{T})$ and $\quad x=\frac{\ln \left(\frac{S e^{-d T}}{X e^{-r T}}\right)}{\sigma \sqrt{T}}+\frac{1}{2} \sigma \sqrt{T}$

These are the general FORMS of the proposal, however, we can use COMBINATORICS to obtain different possible Combination of TERMS and SIGNS (to achieve different values of $G a p_{\text {Stressed }}$ : positive, negative, high, low, close and so on).We can find the OPTIMUM values of GAP OPTIONS CLASS 1 and 2 in the same way as in the case of COMPOUND OPTIONS CLASS 1 and 2 above.

2.4.7 Asset Exchange Options

Class 1:

$$
\begin{aligned}
C_{\text {Stressed }}= & S_{1} e^{-d_{1} T}\left[N\left(\mu_{S_{1}} \mu_{S_{2}} x\right) \pm \sigma_{S_{1}} f_{1}(.) \pm \sigma_{S_{2}} f_{2}(.)\right] \\
& -S_{2} e^{-d_{2} T}\left[N\left(\mu_{S_{1}} \mu_{S_{2}}(x-\Sigma \sqrt{T})\right) \pm \sigma_{S_{1}} f_{1}(.) \pm \sigma_{S_{2}} f_{2}(.)\right]
\end{aligned}
$$

Class 2:

$$
\begin{aligned}
C_{\text {Stressed }}= & S_{1} e^{-d_{1} T}\left[N\left(\mu_{S_{1} S_{2}} x\right) \pm \sigma_{S_{1} S_{2}} f_{1}(.) \pm \sigma_{S_{1} S_{2}} f_{2}(.)\right] \\
& -S_{2} e^{-d_{2} T}\left[N\left(\mu_{S_{1} S_{2}}(x-\Sigma \sqrt{T})\right) \pm \sigma_{S_{1} S_{2}} f_{1}(.) \pm \sigma_{S_{1} S_{2}} f_{2}(.)\right]
\end{aligned}
$$


Where, $C=S_{1} e^{-d_{1} T} N(x)-S_{2} e^{-d_{2} T} N(x-\Sigma \sqrt{T}), \quad x=\frac{\ln \left(\frac{S_{1} e^{-d_{1} T}}{S_{2} e^{-d_{2} T}}\right)}{\Sigma \sqrt{T}}+\frac{1}{2} \Sigma \sqrt{T}$ and $\Sigma=\sigma_{1}^{2}+\sigma_{2}^{2}-2 \rho \sigma_{1} \sigma_{2}$.

These are the general FORMS of the proposal, however, we can use COMBINATORICS to obtain different possible Combination of TERMS and SIGNS (to achieve different values of $C_{\text {Stressed }}$ : positive, negative, high, low, close and so on).We can find the OPTIMUM values of ASSET EXCHANGE OPTIONS CLASS 1 and 2 in the same way as in the case of COMPOUND OPTIONS CLASS 1 and 2 above.

\subsection{Proposed Theorem (Lam's Theorem)}

Let $\left(N\left(d_{i}\right), N_{2}\left(a_{i}, b_{i} ; c_{i}\right), \ldots, N_{n}\left(a_{i}, b_{i}, \ldots, c_{i} ; d_{i}\right)\right), i=1,2, \ldots, n$ such that $n+m \in N$ are the number of terms and $m$ is the number of repeated NORMAL probability type of an Exotic Options pricing model $M_{\text {Normal }}$ whose probabilities are in this form, where $N_{n}\left(a_{i}, b_{i}, \ldots, c_{i} ; d_{i}\right)$ is a Multivariate Normal Distribution. Let $s$ and $f(s, \mu, \sigma, \xi)$ be the underlying stock return andBEST FITTED fat - tailed probability distribution of the underlying stock returns of company $\mathbf{A}$ respectively. Let $y_{1}, y_{2}, \ldots, y_{n}$ be the underlying returns of $M_{\text {Normal }}$ STRIKES (Independent Variables) excluding underlying stock return. Let $f_{1}\left(y_{1}, \mu, \sigma, \pi\right), f_{2}\left(y_{2}, \mu, \sigma, \pi\right), \ldots, f_{n}\left(y_{n}, \mu, \sigma, \pi\right)$ beBEST FITTED fat - tailed probability distribution of the underlying $M_{\text {Normal }}$ STRIKES (Independent Variables). Let $\left(\mu_{y_{1}}, \mu_{y_{2}}, \ldots, \mu_{y_{n}}\right)$ and $\left(\sigma_{y_{1}}, \sigma_{y_{2}}, \ldots, \sigma_{y_{n}}\right)$ be vectors of respective Geometric Return of the Arithmetic Means of the macroeconomic indicators plus research company underlying stock returns (and STRIKES returns) and the respective Geometric volatility of the volatilities of the macroeconomic indicators plus research company underlying stock returns (and STRIKES returns). Define $\mu_{S}$ and $\sigma_{S}$ as above then each of theCOMPONENTS of $\left(N\left(d_{i}\right), N_{2}\left(a_{i}, b_{i} ; c_{i}\right), \ldots, N_{n}\left(a_{i}, b_{i}, \ldots, c_{i} ; d_{i}\right)\right), i=1,2, \ldots, n$ in $M_{\text {Normal }}$ can be contractionally and expansionallySTRESSED using Jameel's stress methods as:

$$
\left(\begin{array}{l}
\left(N\left(\mu_{s} \mu_{y_{1}} \ldots \mu_{y_{n}} d_{i}\right) \pm \sigma_{s} f(s, \mu, \sigma, \xi) \pm \sigma_{y_{1}} f_{1}\left(y_{1}, \mu, \sigma, \pi\right) \pm \ldots \pm \sigma_{y_{n}} f_{n}\left(y_{n}, \mu, \sigma, \pi\right)\right), \\
\left(N_{2}\left(\mu_{s} \mu_{y_{1}} \ldots \mu_{y_{n}}\left(a_{i}, b_{i} ; c_{i}\right)\right) \pm \sigma_{s} f(s, \mu, \sigma, \xi) \pm \sigma_{y_{1}} f_{1}\left(y_{1}, \mu, \sigma, \pi\right) \pm \ldots \pm \sigma_{y_{n}} f_{n}\left(y_{n}, \mu, \sigma, \pi\right)\right), \ldots, \\
\left(N_{n}\left(\mu_{s} \mu_{y_{1}} \ldots \mu_{y_{n}}\left(a_{i}, b_{i}, \ldots, c_{i} ; d_{i}\right)\right) \pm \sigma_{s} f(s, \mu, \sigma, \xi) \pm \sigma_{y_{1}} f_{1}\left(y_{1}, \mu, \sigma, \pi\right) \pm \ldots \pm \sigma_{y_{n}} f_{n}\left(y_{n}, \mu, \sigma, \pi\right)\right)
\end{array}\right), i=1,2, \ldots, n
$$

Optimum at:

$$
\left(\begin{array}{l}
\left(N\left(d_{i}\right) \pm \sigma_{s} f(s, \mu, \sigma, \xi) \pm \sigma_{y_{1}} f_{1}\left(y_{1}, \mu, \sigma, \pi\right) \pm \ldots \pm \sigma_{y_{n}} f_{n}\left(y_{n}, \mu, \sigma, \pi\right)\right), \\
\left(N_{2}\left(a_{i}, b_{i} ; c_{i}\right) \pm \sigma_{s} f(s, \mu, \sigma, \xi) \pm \sigma_{y_{1}} f_{1}\left(y_{1}, \mu, \sigma, \pi\right) \pm \ldots \pm \sigma_{y_{n}} f_{n}\left(y_{n}, \mu, \sigma, \pi\right)\right), \ldots, \\
\left(N_{n}\left(a_{i}, b_{i}, \ldots, c_{i} ; d_{i}\right) \pm \sigma_{s} f(s, \mu, \sigma, \xi) \pm \sigma_{y_{1}} f_{1}\left(y_{1}, \mu, \sigma, \pi\right) \pm \ldots \pm \sigma_{y_{n}} f_{n}\left(y_{n}, \mu, \sigma, \pi\right)\right)
\end{array}\right), i=1,2, \ldots, n
$$

for some $\sigma^{\prime} s=1$ with (i) All $\mu_{S}=\mu_{y_{1}}=\mu_{y_{2}}=\ldots=\mu_{y_{n}}=1$ and (ii) Some $\mu^{\prime} s=1$.

\section{OR}

$$
\left(\begin{array}{l}
\left(N\left(\mu_{s y_{1}} \cdots y_{n} d_{i}\right) \pm \sigma_{s} f(s, \mu, \sigma, \xi) \pm \sigma_{s y_{1}} \cdots y_{n} f_{1}\left(y_{1}, \mu, \sigma, \pi\right) \pm \ldots \pm \sigma_{s y_{1}} \cdots y_{n} f_{n}\left(y_{n}, \mu, \sigma, \pi\right)\right), \\
\left(N_{2}\left(\mu_{s y_{1} \cdots y_{n}}\left(a_{i}, b_{i} ; c_{i}\right)\right) \pm \sigma_{s} f(s, \mu, \sigma, \xi) \pm \sigma_{s y_{1}} \cdots y_{n} f_{1}\left(y_{1}, \mu, \sigma, \pi\right) \pm \ldots \pm \sigma_{s y_{1}} \cdots y_{n} f_{n}\left(y_{n}, \mu, \sigma, \pi\right)\right), \ldots, \\
\left(N_{n}\left(\mu_{s y_{1}} \cdots y_{n} d_{i}\left(a_{i}, b_{i}, \ldots, c_{i} ; d_{i}\right)\right) \pm \sigma_{s} f(s, \mu, \sigma, \xi) \pm \sigma_{s y_{1}} \cdots y_{n} f_{1}\left(y_{1}, \mu, \sigma, \pi\right) \pm \ldots \pm \sigma_{s y_{1}} \cdots y_{n} f_{n}\left(y_{n}, \mu, \sigma, \pi\right)\right)
\end{array}\right), i=1,2, \ldots, n
$$




\section{Optimum at:}

$$
\left(\begin{array}{l}
\left(N\left(d_{i}\right) \pm \sigma_{s} f(s, \mu, \sigma, \xi) \pm \sigma_{s y_{1}} \cdots_{y_{n}} f_{1}\left(y_{1}, \mu, \sigma, \pi\right) \pm \ldots \pm \sigma_{s y_{1}} \cdots y_{n} f_{n}\left(y_{n}, \mu, \sigma, \pi\right)\right), \\
\left(N_{2}\left(a_{i}, b_{i} ; c_{i}\right) \pm \sigma_{s} f(s, \mu, \sigma, \xi) \pm \sigma_{s y_{1}} \cdots y_{n} f_{1}\left(y_{1}, \mu, \sigma, \pi\right) \pm \ldots \pm \sigma_{s y_{1}} \cdots y_{n} f_{n}\left(y_{n}, \mu, \sigma, \pi\right)\right), \ldots, \\
\left(N_{n}\left(a_{i}, b_{i}, \ldots, c_{i} ; d_{i}\right) \pm \sigma_{s} f(s, \mu, \sigma, \xi) \pm \sigma_{s y_{1}} \cdots y_{n} f_{1}\left(y_{1}, \mu, \sigma, \pi\right) \pm \ldots \pm \sigma_{s y_{1}} \cdots y_{n} f_{n}\left(y_{n}, \mu, \sigma, \pi\right)\right)
\end{array}\right), i=1,2, \ldots, n
$$

for some $\sigma_{y_{1}, y_{2}, y_{n}, s}$ 's $s=1$ with (i) All $\mu_{y_{1} y_{2} \ldots y_{n} . S}=1$ and (ii) Some $\mu^{\prime} s=1$. Also, there exists $c, d \in R^{+}$such that $c<d$ then $c<P_{i(\text { Stressed })}^{-} \leq P_{i} \leq P_{i(\text { Stressed })}^{+}<b$ for each $i$.

\subsubsection{Jameel's Credit Risk World Diagram:}

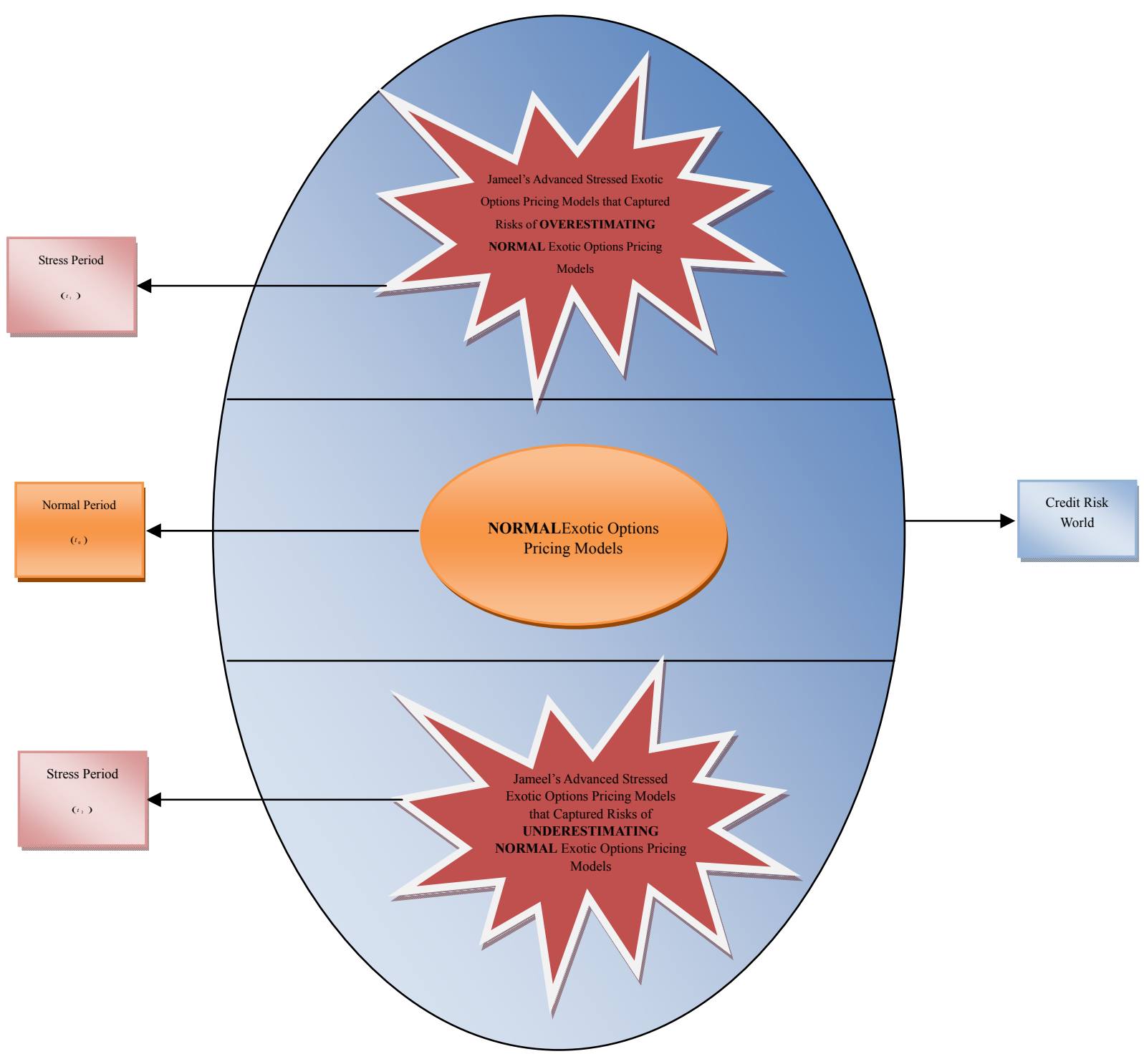

Figure 2. Kano ta Dabo's Diagram 


\section{Result and Discussion}

All the proposed Jameel's Advanced Stressed Exotic Options Pricing Models are expected to adequately and efficiently work (more especially at the times of economic and financial recoveries or recessions) at their OPTIMUM VALUES stated in each case of the proposals based on the Jameel's Criterion and numerical results established and published in my previous papers.More so, the Models are expected to dramatically increases the markets CONFIDENCE and drastically decreases the markets RISKS.Jameel's Advanced Stressed Exotic Options Pricing Methods can be directly applied in other stochastic fields of SCIENCES, ARTS, SOCIAL AND MANAGEMENT SCIENCES, MEDICINE AND ENGINEERING to capture chaotic situations or to increase the power of the existing models' sensitivities to change.

\section{Conclusion}

The existing Exotic options pricing models underestimates (overestimates) prices especially at the times of economic and financial recoveries or recessions just because of their simple Normality Assumption and failure to incorporate underlying assets returns probability distributions. However, based on the research findings, the author improved the existing models by incorporating fat - tailed effects of the underlying assets returns of exotic derivative product in question using Jameel's Criterion and Jameel'sContractional and Expansional Stress Methods to enable them capture potential black swans.

For the sake of practitioners, it is believe that the existing exotic options pricing models out rightly underestimates (overestimates) exotic products prices especially at the stress periods to extent in which Nassim N. Taleb and EspenGararder Hang (2011) wrote a paper entitled: ' Option Traders use (very) Sophisticated Heuristic, never the Black - Scholes - Merton Formula', they stated that the formula is 'fragile to jumps and tail events' just because of its Normality Assumption thereby it is one of the major factors that contributed to the late $2007-2008$ economic and financial crises (thanks to the Black - Scholes - Merton generous invention). In view of the above, all the proposed Jameel's Advanced Stressed exotic options pricing models class 1 and 2 presented in this paper, will be more reliable, robust, sophisticated, holistic and extraordinary, providing better approximations, increasing the probabilities of high losses and above all have the ability to precisely traces the trajectories of the past and future economic and financial crises related to exotic options pricing.

Finally, for the sake of future research direction, the models can be improved further to capture more vital information using more macroeconomic indicators and models' independent variables than ordinarily only $S, X_{1}$ and $X_{2}$ or $S, K_{1}$ and $K_{2}$.

CreditMetrics" (1997) stated that "We remind our readers that no amount of sophisticated analytics will replace experience and professional judgment in managing risks. CreditMetrics ${ }^{\mathrm{TM}}$ is nothing more than a high-quality tool for the professionalrisk manager in the financial markets and is not a guarantee of specific results."

"If a seatbelt does not provide perfect protection, it still makes sense to wear one, it is better to wear a seatbelt than to not wear one". It is better off improving Exotic Options PricingModels to incorporate fat - tailed effects than not.

\section{References}

Basel Committee on Banking Supervision (2006). International Convergence of Capital Standards. A Revised Framework Comprehensive Version, Bank for International Settlement, CH - 4002 Basel, Switzerland

Charles Smithson et al. (2000). How the Market Values Credit Derivatives. Journal of Lending \& Credit Risk Management, March, 1-3.

Gerald W. Buetow, Jr. (1999). Ratchet Options. Journal of Financial and Strategic Decisions, 12(2), 19-22.

J P Morgan. (2009). The J.P. Morgan Guide to Credit Derivatives, with Contributions. from the RiskMetrics Group, Published by Risk, 25, 744-759

Jamilu A. Adamu. (2013). A Guide to Financial Mathematics and Risk Management for Nigeria ( $1^{\text {st }}$ Ed.). Delcon Press, Suleja, Niger.

Jamilu A. Adamu. (2014). Modern Approach to Financial Risk Management( $1^{\text {st }}$ Ed.). Delcon Press: Suleja, Niger.

Jamilu A. Adamu. (2014). Understanding Financial Risks( $1^{\text {st }}$ Ed.). Delcon Press: Suleja, Niger. 
Jamilu Auwalu Adamu. (2015). Banking and Economic Advanced Stressed Credit Rating Models. submitted for Publication the Risk Journals, Credit Risk Journal.

Jamilu Auwalu Adamu. (2015). Banking and Economic Advanced Stressed Probability of Default Models. Asian Journal of Management Sciences, 3(8), 10-18.\#

Jamilu Auwalu Adamu. (2015). Global Economic and Financial Crises Advanced Stressed Derivatives Pricing Models. Asian Journal of Management Sciences, 3(10), 11-24.

Jamilu Auwalu Adamu. (2015). Global Economic and Financial Crises Best Fitted Fat - Tailed Effects Probability Distributions. submitted for Publication in the Asian Journal of Management Sciences.

Jian Wu \& Wei Yu. (2010). How to Classify Existing Exotic Options and Design New Ones. An International Journal of Business \& Economics, 5(1), Fall 2010.

Les Clewlow et al. (1994). Pricing Exotic Options in a Black - Scholes World. Financial Options Research Centre, Warwick Business University of Warwick Coventry CV4 7AL, FORC preprint: 94/54.

Nassim N. Taleb \& Espen Gararder Hang. (2011). Option Traders use (very) Sophisticated Heuristic, never the Black - Scholes - Merton Formula. Journal of Economic Behavior \& Organization, 77, 97-106. http://dx.doi.org/10.1016/j.jebo.2010.09.013

Nassim N. Taleb et al. (2009). Risk Externalities and Toobid to Fail. New York University Polytechnic Institute, 11201, New York, United States.

Nassim N. Taleb. (2007). Black Swans and the Domains of Statistics. American Statistician, 6I(3), 1-3.

Nassim N. Taleb. (2012). The Illusion of Thin - Tails under Aggregation. NYU - Poly, January, 2012.

Professionals Risk Management International Association (PRMIA) Handbook, 2003 and 2010

Raimonda Martinkute - Kauliene (2012). Exotic Options: A Chooser Option and its Pricing. Business, Management and Education, 10(2), 289-301. http://dx.doi.org/10.3846/bme.2012.20

Robert Geske (1979). The Valuation of Compound Options. Journal of Financial Economics, 7, 63-81. http://dx.doi.org/10.1016/0304-405X(79)90022-9

Sandica Ana - Maria. (2010). Credit Scoring Modelling: A Micro - Macro Approach. Academy of Economic Studies, Doctoral School of Finance DOFIN, 2010. 